On the boundary layer arising in the spin-up of a stratified fluid in a container with sloping walls

Duck, P.W. and Foster, M.R. and Hewitt, R.E.

1997

MIMS EPrint: 2010.87

Manchester Institute for Mathematical Sciences

School of Mathematics

The University of Manchester

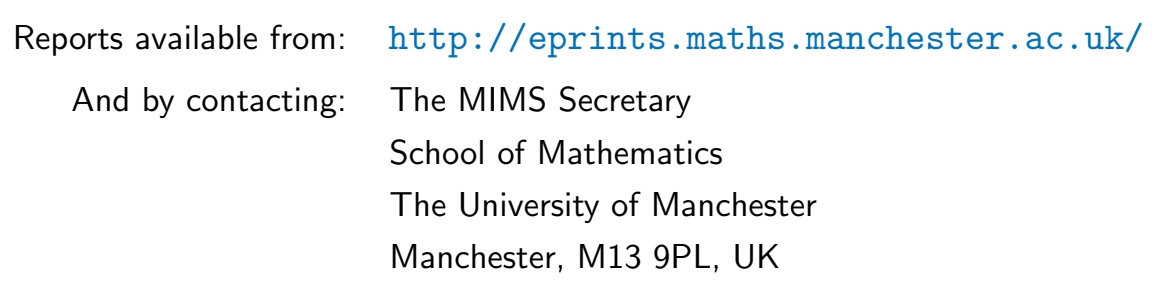

ISSN 1749-9097 


\title{
On the boundary layer arising in the spin-up of a stratified fluid in a container with sloping walls
}

\author{
By P. W. DUCK ${ }^{1}$, M. R. FOSTER AND R. E. HEWITT $T^{1} \dagger$ \\ ${ }^{1}$ Department of Mathematics, University of Manchester, \\ Oxford Road, Manchester M13 9PL, UK \\ 2 Department of Aerospace Engineering, Applied Mechanics and Aviation, \\ The Ohio State University, Columbus, OH, 43210, USA
}

(Received 13 December 1995 and in revised form 24 October 1996)

In this paper we consider the boundary layer that forms on the sloping walls of a rotating container (notably a conical container), filled with a stratified fluid, when flow conditions are changed abruptly from some initial (uniform) state. The structure of the solution valid away from the cone apex is derived, and it is shown that a similarity-type solution is appropriate. This system, which is inherently nonlinear in nature, is solved numerically for several flow regimes, and the results reveal a number of interesting and diverse features.

In one case, a steady state is attained at large times inside the boundary layer. In a second case, a finite-time singularity occurs, which is fully analysed. A third scenario involves a double boundary-layer structure developing at large times, most significantly including an outer region that grows in thickness as the square-root of time.

We also consider directly the nonlinear fully steady solutions to the problem, and map out in parameter space the likely ultimate flow behaviour. Intriguingly, we find cases where, when the rotation rate of the container is equal to that of the main body of the fluid, an alternative nonlinear state is preferred, rather than the trivial (uniform) solution.

Finally, utilizing Laplace transforms, we re-investigate the linear initial-value problem for small differential spin-up studied by MacCready \& Rhines (1991), recovering the growing-layer solution they found. However, in contrast to earlier work, we find a critical value of the buoyancy parameter beyond which the solution grows exponentially in time, consistent with our nonlinear results.

\section{Introduction and formulation}

Unsteady adjustments to new conditions in various geophysical settings take place in ways that are dependent on the character of the layers bounding the region of interest. Greenspan \& Howard (1963) have described how such adjustments occur in a homogeneous rotating fluid: spin-up, or other adjustment, is achieved over a time scale of order $h /(v \Omega)^{1 / 2}$, where $v$ is the kinematic viscosity coefficient, $\Omega$ is the rotation rate of the frame of reference and $h$ is some vertical scale of the container. (Although such a scale is important for analysis of spin-up, it is not essential for the analysis of the boundary layer.) On the other hand, when stratification is present, a

$\dagger$ Present address: Department of Civil Engineering, The University, Dundee DD1 4HN, UK. 


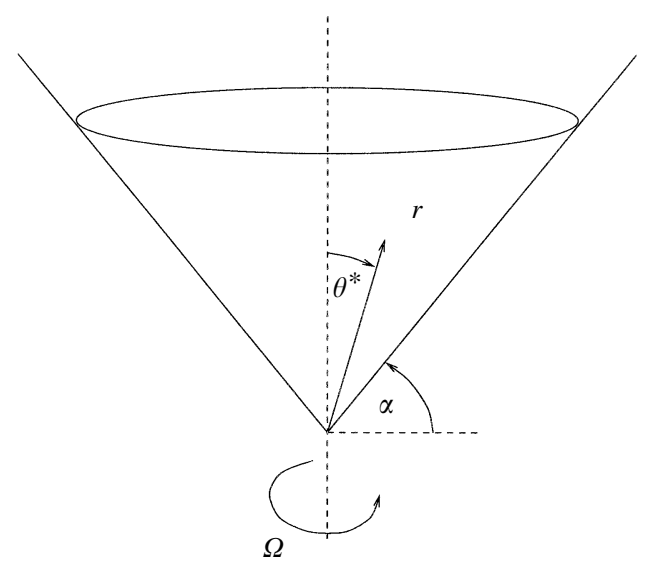

FIGURE 1. Geometrical configuration for the flow.

series of papers (see Walin 1969 and also Spence, Foster \& Davies 1992 for a brief review and some new results) confirm that the unsteady adjustments occur over a much longer time scale, namely a diffusive scale. All these results are for containers without sloping walls.

Because there is a component of buoyancy along a solid boundary whose normal is not vertical, the character of any boundary layers is altered dramatically if the walls are sloping. Thorpe (1987) has obtained a (linearized) solution for a steady slopingwall boundary layer, which can apparently be fitted into an overall steady-state solution under very special circumstances only. MacCready \& Rhines (1991), in the case of a planar wall, have shown that for a general situation such a boundary layer does not develop towards a steady state for long times. Hence, spin-up of rotating stratified fluids in containers with sloping bounding walls will be quite different from similar unsteady problems in containers for which walls are horizontal and vertical.

In this paper, we explore further the unsteady nature of the boundary layer on a sloping wall, confirming and extending what was found by MacCready \& Rhines (1991), but adding some details to the understanding of the double-structured nature of the boundary layer, resolving the question of the relevance of the Thorpe (1987) solution. In particular, we study the boundary layer on the conical surface of a container of stratified fluid, as shown in figure 1. We determine in some detail the character of the boundary-layer solutions in the nonlinear regime, when changes in the rotation rate are large (the previous work in this area has focused on the linear regime, corresponding to small changes in rotation rate).

In this first instance, it is simplest to work in an inertial (non-rotating) frame of reference, in spherical polar coordinates. Let $\left(v_{r}, v_{\theta}, v_{\phi}\right)$ be velocity components in the $\left(r, \theta^{\star}, \phi\right)$ directions, where $\theta^{\star}$ is the polar angle, and $\phi$ is azimuthal (see figure 1$)$. The density is decomposed in the form $\rho_{o}+\bar{\rho}(z)+\rho^{\prime}$, where $|\bar{\rho}|,\left|\rho^{\prime}\right| \ll \rho_{o}$. MacCready \& Rhines (1991) write $B=g \rho^{\prime} / \rho_{o}$, where $\rho^{\prime}$ is the baroclinic density perturbation on the linear stratification, $\rho_{o}$ is a reference density and $g$ is gravity. Here, it is convenient to use a dimensionless version of their $B$, namely $B_{M R} /\left(\Omega^{2} h\right) \equiv B$. Making lengths dimensionless with a characteristic scale of the container, say $h$, let the boundary-layer coordinate $\theta$ be defined by $\theta^{\star}-(\pi / 2-\alpha) \equiv\left(v / \Omega h^{2}\right)^{1 / 2} \theta$, where $\alpha$ is the angle of the sloping wall relative to the horizontal. The velocity components are dimensionless with respect to $\Omega h$. Boussinesq equations, under a boundary-layer approximation on 
this sloping wall, then become

$$
\begin{gathered}
\frac{1}{r^{2}} \frac{\partial\left(r^{2} v_{r}\right)}{\partial r}+\frac{1}{r} \frac{\partial v_{\theta}}{\partial \theta}=0 \\
\frac{\partial v_{r}}{\partial t}+(\boldsymbol{q} \cdot \nabla) v_{r}-\frac{v_{\phi}^{2}}{r}+\frac{\mathrm{d} p}{\mathrm{~d} r}=\frac{1}{r^{2}} \frac{\partial^{2} v_{r}}{\partial \theta^{2}}-B \sin \alpha, \\
\frac{\partial v_{\phi}}{\partial t}+(\boldsymbol{q} \cdot \nabla) v_{\phi}+\frac{v_{\phi} v_{r}}{r}=\frac{1}{r^{2}} \frac{\partial^{2} v_{\phi}}{\partial \theta^{2}} \\
\frac{\partial B}{\partial t}+(\boldsymbol{q} \cdot \nabla) B-S v_{r} \sin \alpha=\frac{1}{\sigma} \frac{1}{r^{2}} \frac{\partial^{2} B}{\partial \theta^{2}} \\
\boldsymbol{q} \cdot \nabla \equiv v_{r} \frac{\partial}{\partial r}+\frac{v_{\theta}}{r} \frac{\partial}{\partial \theta}
\end{gathered}
$$

In these equations, $v_{\theta}$ has been scaled in the usual way for a boundary-layer approximation and the pressure, $p$, is that from the outer flow, since the $\theta$-direction equation indicates that pressure is unchanged across the layer. A Burger number is written here as $S \equiv N^{2} / \Omega^{2}$, where $N=\left[-\left(g / \rho_{o}\right)(\partial \bar{\rho} / \partial z)\right]^{1 / 2}$ is the Brunt frequency, which is assumed to be constant. Clearly the time has been scaled with $\Omega^{-1}$, and $\sigma$ is the Schmidt number, which is of the order of 1000 for a solution of salt in water.

These equations are to be solved subject to some initial conditions, some prescribed edge pressure gradient, and appropriate boundary conditions on the velocity components, which in our case are

$$
v_{r}=0, \quad v_{\theta}=0, \quad v_{\phi}=r \omega(t) \quad \text { on } \quad \theta=0,
$$

where $\omega$ is the prescribed angular velocity of the wall, different from the initial angular velocity of the fluid $\Omega$. Requiring that the net flux of density (salinity, in this case) to the wall is zero, a condition appropriate to attempting to describe laboratory results, leads to the final condition on $\theta=0$,

$$
\frac{1}{r} \frac{\partial B}{\partial \theta}=-S \cos \alpha E^{1 / 2}, \quad \text { where } \quad E \equiv \frac{v}{\Omega h^{2}},
$$

$E$ being the Ekman number, which will be assumed to be small throughout this paper. It is interesting to note that, in regard to an asymptotic expansion of the solution in powers of the Ekman number, this latter boundary condition is less important than the dynamical condition, for $\omega-\Omega=O(1)$.

In the case $\alpha \equiv 0$, the equations completely decouple, so that the velocity boundary layer is independent of the buoyancy layer. The steady velocity-layer solution for a spin-up from rest $\left(\omega=1, v_{\phi_{e d g e}}=0\right)$ is, of course, due to von Kármán (1921). The corresponding steady solution for a spin-down to rest $\left(\omega=0, v_{\phi_{\text {edge }}}=r\right)$ is due to Bödewadt (1940). Bodonyi (1978) suggested, after an analysis based upon a computational initial-value problem, that both the von Kármán and Bödewadt solutions approach their respective steady states after long times. We shall discuss the unsteady Bödewadt problem briefly in the final section of this paper, $\S 8$. There is no indication in Bodonyi's, or predecessors' work (see Bodonyi 1978 for a discussion) of either continuously growing solutions or the finite-time breakdown described later in this paper. Interestingly however, Bodonyi \& Stewartson (1977) did find a finitetime breakdown of the initial-value problem for a counter-rotating situation, but the scalings involved are quite different from those found in this work.

The outline of the paper is as follows. In $\$ 2$ we derive the boundary-layer equations 
relevant for $r \gg E^{1 / 2}$, obtaining numerical solutions to the self-similar set in $\S 3$, and further discussing the ultimate behaviour of the solutions in $\S 4$. In $\S 5$ we describe the likely extent, in parameter space, of the different flow regimes. In $\S 6$ the limit of large Schmidt number $(\sigma)$ is considered. In $\S 7$ we return to the linearized version similar to that considered by MacCready \& Rhines (1991), reviewing a few details from their paper, and clarifying the relevance of the Thorpe (1987) solution discussed briefly above. We also find confirmation of one of the important features of the solution parameter space discussed in $\$ 5$ when considering the linearized solution. Our conclusions are given in $\S 8$.

\section{The flow away from the apex}

We use the spherical polar coordinate system and non-dimensionalization defined in the previous section. We suppose that at time $t=0-$ the flow is in some initial state, and at $t=0+$, an abrupt change is introduced into the flow, such as an instantaneous change in the rotation rate of the fluid container (the particular situation we concentrate on in this paper, although other impulsive changes are also acceptable).

On account of the rotational nature of the container walls, reflected in condition (1.6), we expect that if $r \gg E^{1 / 2}$ (and we take $E \ll 1$ throughout) a self-similar solution exists of the form

$$
v_{\phi}=r \hat{W}(\Theta, t)+\ldots,
$$

where $\Theta$ is a scaled and shifted $\theta$-coordinate. Now, a sensible balancing of terms leads to the important scaling

$$
\Theta=r \theta=O(1),
$$

together with

$$
\begin{gathered}
v_{r}=r \hat{U}(\Theta, t)+\ldots, \\
v_{\theta}=\hat{V}(\Theta, t)-\Theta \hat{U}(\Theta, t)+\ldots, \\
p=r^{2} \hat{P}(t)+\ldots, \\
B=r \hat{B}(\Theta, t)+\ldots
\end{gathered}
$$

The additional term in the $v_{\theta}$-expansion leads to 'tidier' resulting equations, whilst not influencing the boundary conditions. The continuity equation (1.1), to leading order in $r$, leads to

$$
2 \hat{U}+\hat{V}_{\Theta}=0,
$$

whilst the momentum equations and the equation of density transport, (1.2)-(1.4), lead to

noting that

$$
\begin{gathered}
\frac{\partial \hat{U}}{\partial t}+\hat{U}^{2}+\hat{V} \hat{U}_{\Theta}-\hat{W}^{2}=-2 \hat{P}+\hat{U}_{\Theta \Theta}-\hat{B} \sin \alpha, \\
\frac{\partial \hat{W}}{\partial t}+2 \hat{U} \hat{W}+\hat{V} \hat{W}_{\Theta}=\hat{W}_{\Theta \Theta}, \\
\frac{\partial \hat{B}}{\partial t}+\hat{V} \hat{B}_{\Theta}-S \sin \alpha \hat{U}+\hat{U} \hat{B}=\frac{1}{\sigma} \hat{B}_{\Theta \Theta},
\end{gathered}
$$

$$
\frac{\partial \hat{P}}{\partial \Theta}=0 .
$$


Since we propose to solve an initial-boundary-value problem, throughout all the differing cases discussed in the subsequent sections of this paper, we will take the boundary conditions at the wall to be given by those derivable from (1.6) and (1.7), therefore

$$
\hat{W}=1, \quad \hat{U}=0, \quad \hat{V}=\frac{\partial \hat{V}}{\partial \Theta}=\frac{\partial \hat{B}}{\partial \Theta}=0 \quad \text { on } \quad \Theta=0 \quad \text { for } \quad t>0,
$$

where we have assumed the rotation rate of the container at $t=0+$ is utilized in the non-dimensionalization process, thus making $\omega$ in (1.6) identically unity. The condition on $\hat{B}_{\Theta}$ arises because this quantity is in fact $O\left(E^{1 / 2} / r\right)$, and is negligible to leading order if $r \gg E^{1 / 2}$. As $\Theta \rightarrow-\infty$

$$
\hat{U} \rightarrow 0, \quad \hat{W} \rightarrow \hat{W}_{e}, \quad \hat{B} \rightarrow \hat{B}_{e}=\frac{\hat{W}_{e}^{2}-2 \hat{P}}{\sin \alpha},
$$

where $\hat{W}_{e}$ denotes the outer edge value of $\hat{W}$, and $\hat{P}$ is the pressure gradient coefficient. The latter condition arises as a result of taking the limit of (2.8) as $\Theta \rightarrow-\infty$. Here we have implicitly relaxed the condition that $\hat{B} \rightarrow 0$ as $\Theta \rightarrow-\infty$ (cf. Thorpe 1987; MacCready \& Rhines 1991) in order to investigate a wider class of solutions to the boundary-layer equations; there appears no reason inherent in the boundary-layer formulation for supposing that $\hat{B}=0$ at the outer edge of the boundary layer. Non-zero $\hat{B}_{e}$ corresponds to the existence of an interior density perturbation; we hasten to point out, however, that such vanishing of $\hat{B}$ at the layer edge is likely the proper condition for analysing the spin-up from rest problem.

The number of parameters in the problem may be reduced by two in the following way. If we write

$$
\begin{aligned}
& B^{*}=2 \hat{P}+\hat{B} \sin \alpha, \\
& S^{*}=S \sin ^{2} \alpha+2 \hat{P},
\end{aligned}
$$

then (2.7) and (2.9) remain unchanged, and (2.8) and (2.10) become

$$
\begin{aligned}
& \frac{\partial \hat{U}}{\partial t}+\hat{U}^{2}+\hat{V} \hat{U}_{\Theta}-\hat{W}^{2}=\hat{U}_{\Theta \Theta}-B^{*}, \\
& \frac{\partial B^{*}}{\partial t}+\hat{V} B_{\Theta}^{*}-S^{*} \hat{U}+\hat{U} B^{*}=\frac{1}{\sigma} B_{\Theta \Theta}^{*} .
\end{aligned}
$$

Under this transformation, the boundary conditions (2.12) and (2.13) become

$$
\hat{W}=1, \quad \hat{U}=0, \quad \hat{V}=0, \frac{\partial B^{*}}{\partial \Theta}=0 \quad \text { on } \quad \Theta=0, \quad t>0,
$$

and

$$
\hat{U} \rightarrow 0, \quad \hat{W} \rightarrow \hat{W}_{e}, \quad B^{*} \rightarrow B_{e}^{*}=\hat{W}_{e}^{2} \quad \text { as } \quad \Theta \rightarrow-\infty .
$$

This system is formally the same as (2.7)-(2.10), (2.12), (2.13), but with $\hat{P}=0$ and $\alpha=\pi / 2$. With this change of variable, the solutions to the boundary-layer equations are characterized by just three independent parameters: a buoyancy parameter, or 'Burger number', $S^{*}$; an edge-to-surface velocity ratio, $\hat{W}_{e}$; and the Prandtl (or Schmidt) number, $\sigma$. In this paper we explore the dependence of the solutions on these three quantities, with the restrictions $S^{*}>0, \hat{W}_{e}>0$. Note that $S^{*}=\hat{W}_{e}^{2}$ corresponds to the non-stratified case, attained by formally taking $S \rightarrow 0, \hat{B} \rightarrow 0$.

Throughout the analysis, we will suppose that at $t=0-$ the fluid in the boundary layer takes the conditions at the edge and that the wall itself moves with the speed 
of the fluid; all problems discussed herein involve an impulsive change in the wall velocity at $t=0+$. Hence

$$
\hat{U}=0, \quad \hat{V}=0, \quad B^{*}=B_{e}^{*}, \quad \hat{W}=\hat{W}_{e} \quad \text { for all } \quad \Theta \quad \text { at } t=0-.
$$

The impulsive nature of the boundary conditions at $t=0+$ must lead to a singular solution at $t=0$, with, in particular, the formation of a sublayer, investigated below.

\section{Short-time behaviour}

Let us consider the nature of the solution of the system (2.7), (2.9), (2.16)-(2.19) as $t \rightarrow 0$. Inspection of the system immediately suggests that $\Theta=O\left(t^{1 / 2}\right)$ is the key (inner) scale. The nature of the azimuthal velocity on the wall also demands that $\hat{W}=O(1)$, whilst the governing equations demand that $B^{*}=O(1), \hat{V}=O\left(t^{3 / 2}\right)$ on this scale.

We therefore write

$$
\begin{gathered}
\hat{W}=\bar{W}(\eta)+\ldots, \\
B^{*}=\bar{B}(\eta)+\ldots, \\
\hat{V}=t^{3 / 2} \bar{V}(\eta)+\ldots,
\end{gathered}
$$

with

$$
\eta=\Theta / t^{1 / 2}
$$

Substitution of the above into (2.7), (2.9), (2.16)-(2.19) and taking leading-order terms in $t$, leads to the following system:

$$
\begin{gathered}
\sigma \bar{B}_{\eta \eta}+\frac{1}{2} \eta \bar{B}_{\eta}=0, \\
\bar{W}_{\eta \eta}+\frac{1}{2} \eta \bar{W}_{\eta}=0, \\
\bar{V}_{\eta \eta \eta}+\frac{1}{2} \eta \bar{V}_{\eta \eta}-\bar{V}_{\eta}-2 \bar{W}^{2}+2 \bar{B}=0 .
\end{gathered}
$$

The boundary conditions reduce to

$$
\bar{B}_{\eta}=\bar{V}_{\eta}=\bar{V}=0, \quad \bar{W}=1 \quad \text { on } \quad \eta=0,
$$

together with

$$
\bar{W} \rightarrow \Omega, \quad \bar{B} \rightarrow \bar{B}_{-}, \bar{V} \rightarrow \frac{1}{2} \eta^{2} \hat{V}_{\Theta \Theta}(\Theta=0, t=0-) \quad \text { as } \quad \eta \rightarrow-\infty,
$$

where

$$
\bar{B}_{-}=\hat{B}(0,0-),
$$

and

Consequently we see

$$
\Omega=\hat{W}(0,0-) .
$$

$$
\begin{gathered}
\hat{W}=\frac{1-\Omega}{\pi^{1 / 2}} \int_{0}^{\eta} \mathrm{e}^{-\eta^{2} / 4} \mathrm{~d} \eta+1, \\
\bar{B}=\hat{B}_{-},
\end{gathered}
$$

whilst the solution of (2.27) is not stated so simply, but is nonetheless straightforward to evaluate (at least numerically). The outer solution, namely that for $\Theta=O(1)$, takes the form

$$
\hat{W}=W_{0}(\Theta)+t^{1 / 2} W_{1}(\Theta)+\ldots,
$$




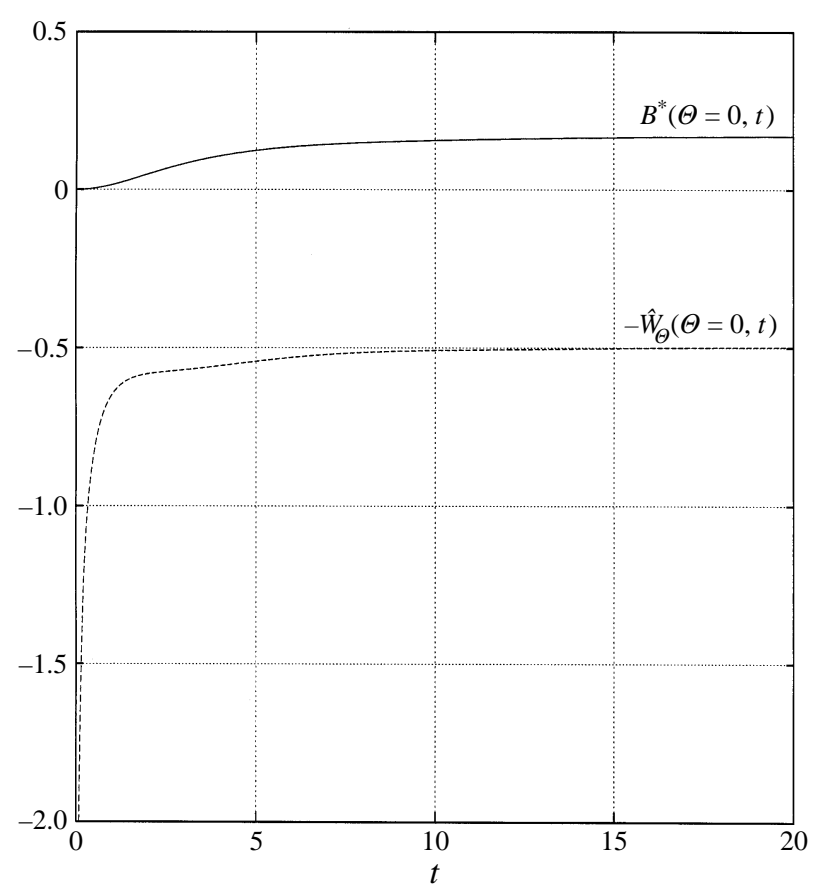

FIGURE 2. Temporal development of $B^{*}(\Theta=0)$ and $-\hat{W}_{\Theta}(\Theta=0)$, pure spin-up case

$$
\begin{gathered}
\left(\hat{W}_{e}=\hat{B}_{e}=0, S^{*}=0.5\right) . \\
\hat{V}=V_{0}(\Theta)+t^{1 / 2} V_{1}(\Theta)+\ldots, \\
B^{*}=B_{0}(\Theta)+t^{1 / 2} B_{1}(\Theta)+\ldots,
\end{gathered}
$$

where $W_{0}, V_{0}, B_{0}$ denote the values of $\hat{W}, \hat{V}, \hat{B}$ at $t=0-$. The solution for $W_{1}, V_{1}, B_{1}$ depends on $W_{0}, V_{0}, W_{0}$ and is routine, and will not be considered here.

In the following section, we present some numerical results that reveal a number of interesting phenomena.

\section{Numerical results}

Two numerical schemes, one explicit in $t$ (first-order time accurate), the other implicit in $t$ (second-order time accurate), both using a central-difference scheme in $\Theta$ (second-order accurate) were implemented to solve the system (2.7), (2.9), (2.16)(2.19). There are a variety of subclasses to the problem, and we consider a number of these (which illustrate several diverse characteristics) in turn. Note that in all cases considered in this paper, unless otherwise stated (as in $\$ 6$ and $\S 7$, for example), we have chosen $\sigma=1$. We find that there are three distinct long-time states for spin-up or spin-down of the container, depending on the relative sizes of $S^{*}, \hat{W}_{e}$ and $\sigma$. A typical result for each case is given below.

\section{(i) Steady state}

We consider first an example of a spin-up from rest problem; the initial conditions are as indicated in (2.20), and boundary conditions are described by (2.18) and (2.19) with

$$
\hat{W}_{e}=0, \quad B_{e}^{*}=0, \quad S^{*}=\frac{1}{2} .
$$



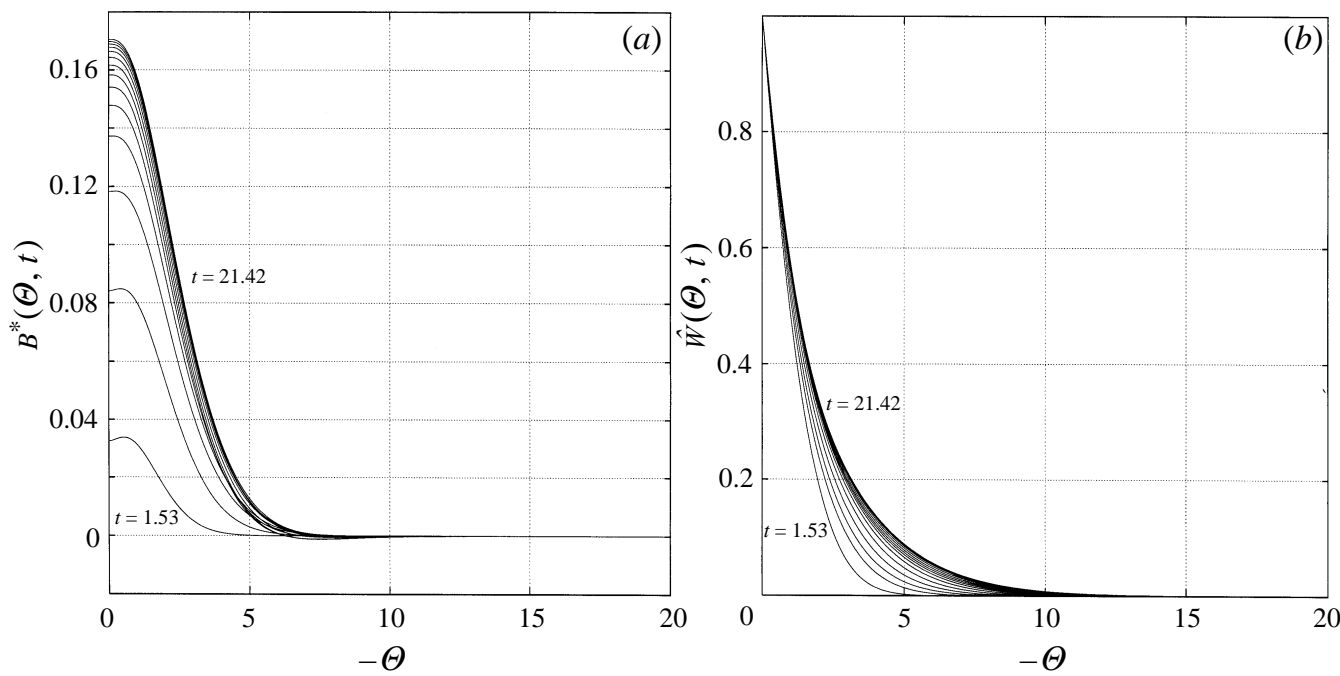

Figure 3. Profiles of $(a) B^{*}$, and $(b) \hat{W}$, pure spin-up case $\left(\hat{W}_{e}=\hat{B}_{e}=0, S^{*}=0.5\right)$.

The temporal development of $B^{*}(0, t)$ and $\hat{W}_{\Theta}(0, t)$ in this case is shown in figure 2. It is quite clear that both the above quantities asymptote to a steady limit as $t$ increases. This is confirmed by the distributions of $B^{*}(\Theta)$ and $\hat{W}(\Theta)$ at selected values of $t$ (taken at equal intervals), shown in figures $3(a)$ and $3(b)$ respectively. Consequently, the ultimate structure of the flow would appear to be merely the system (2.7), (2.9), (2.16)-(2.19), with the time-derivative terms omitted.

In the following examples we consider cases for which the rotational speed of the conical wall changes abruptly, at $t=0$, from $\hat{W}_{e}$ to unity, which might involve either spin-up or spin-down. Other initial and boundary values are as indicated in (2.18)-(2.20), and in particular,

$$
\begin{gathered}
\hat{W}_{e}>0, \\
B_{e}^{*}=\hat{W}_{e}^{2} .
\end{gathered}
$$

\section{(ii) Finite-time singularity}

Consider first spin-down from an initial state $\left(\hat{W}_{e}>1\right)$, with $S^{*}=\frac{1}{2}$. Results for $B^{*}(0, t)$ and $\hat{W}_{\Theta}(0, t)$ for the particular choice $\hat{W}_{e}=1.5$, are shown in figure 4 . Intriguingly it appears that both of these quantities exhibit rapid growth, indicating the existence of a singularity, at some finite time, so therefore no steady state is achieved. This behaviour is suggestive of a catastrophic breakdown of the boundary layer, presumably linked to some form of eruption. The nature of this singularity is analysed in detail in $\$ 4.2$ below. We surmise that the breakdown is caused by the general retardation of the boundary layer, coupled with buoyancy effects.

(iii) Growing boundary layer

A third scenario is possible, as shown in a second spin-down example: for $\hat{W}_{e}=1.5$, $S^{*}=2.75$, results for $B^{*}(0, t)$ and $\hat{W}_{\Theta}(0, t)$ are shown in figure 5. Although these results suggest steady values are approached as $t \rightarrow \infty$ (with $\hat{W}_{\Theta}(0, t) \rightarrow 0$ ), figures $6(a)$ and $6(b)$, which show distributions of $B^{*}(\Theta, t)$ and $\hat{W}(\Theta, t)$, present a contrasting 


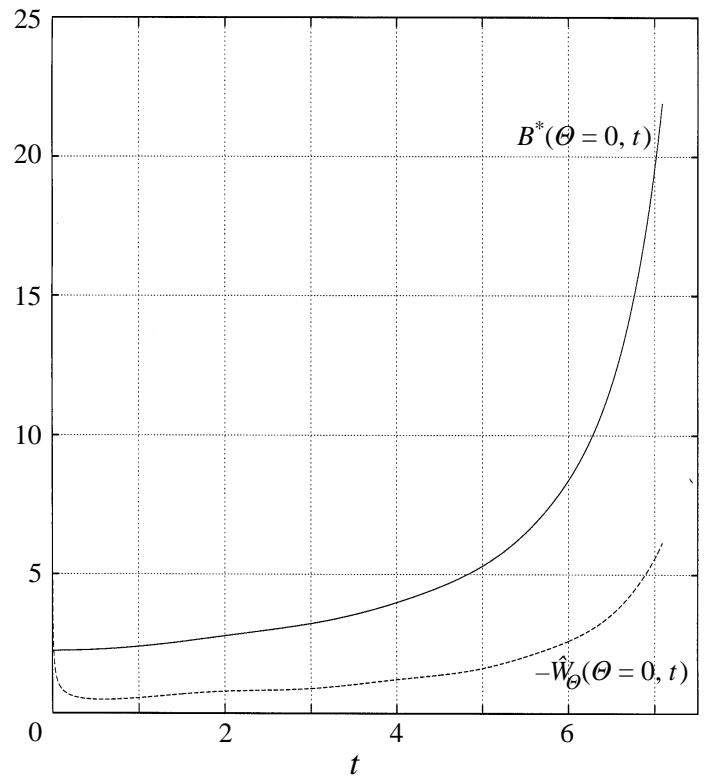

FIGURE 4. Temporal development of $B^{*}(\Theta=0)$ and $-\hat{W}_{\Theta}(\Theta=0), S^{*}=0.5, \hat{W}_{e}=1.5$.

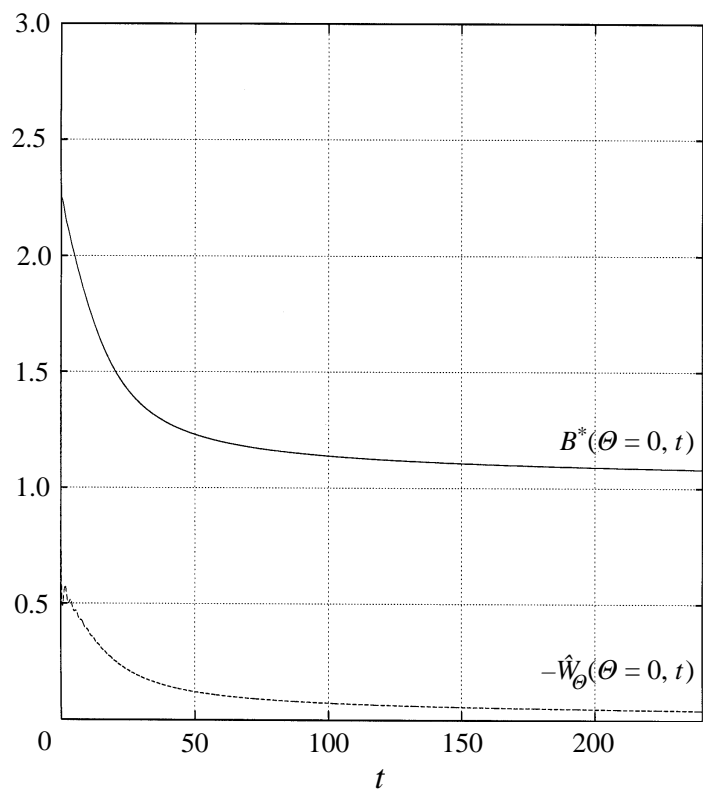

FIGURE 5. Temporal development of $B^{*}(\Theta=0)$ and $-\hat{W}_{\Theta}(\Theta=0), S^{*}=2.75, \hat{W}_{e}=1.5$.

picture. It appears that a thickening of the boundary layer is occurring, rather than a fully steady state being approached as time increases.

Numerous other examples were computed by the authors (in the range $\hat{W}_{e} \geqslant 0$ ), and all calculations fell into one of the categories described above. The detailed structure of each of these three distinct $t \rightarrow \infty$ behaviours is given in the next section. 

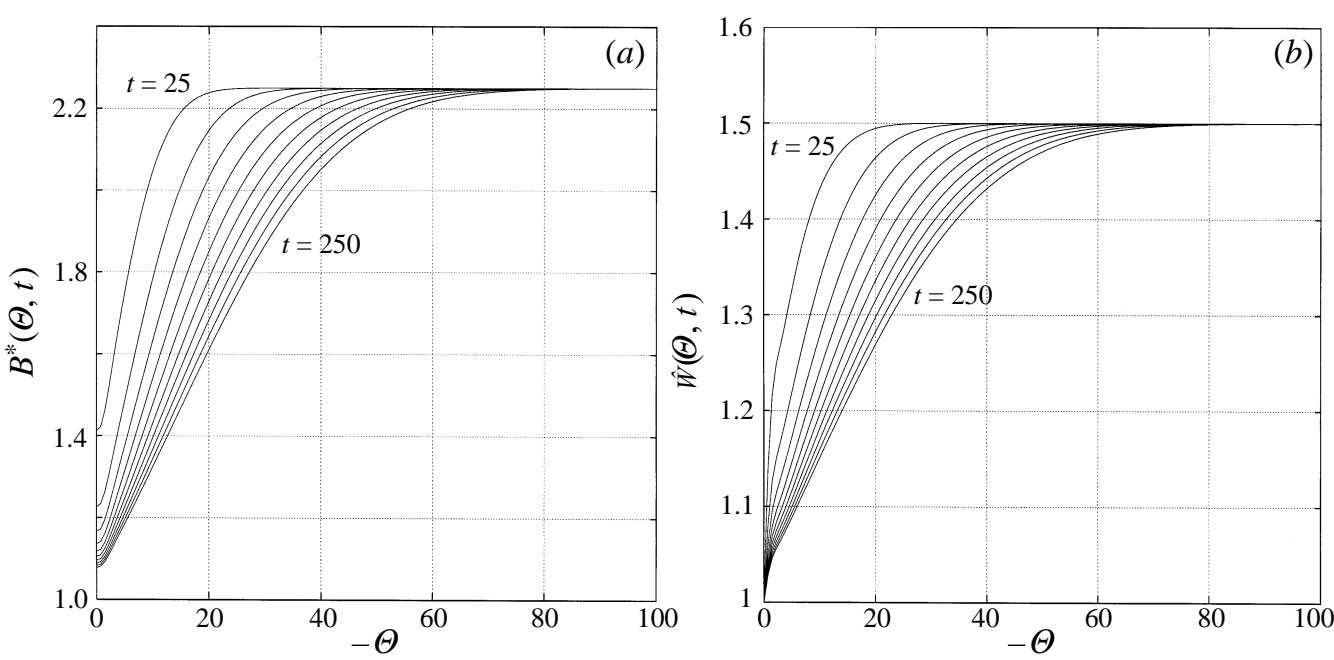

FIgURE 6. Profiles of $(a) B^{*}$ and $(b) \hat{W}: S^{*}=2.75, \hat{W}_{e}=1.5$.

\section{Mathematical description of the ultimate behaviour of the flow}

From the sample results of the previous section, there are three distinct possibilities for the terminal state of the flow. The first of these, the attainment of a true steady state, appears straightforward, as pointed out previously. However the other two cases require some investigation, namely the case in which a growing boundary layer occurs and the case for which a catastrophic failure of the boundary-layer solution arises. We deal with these possible scenarios below. In all of the analysis of this section, we take $\sigma=O(1)$; we consider the case of extreme values of $\sigma$ in $\S 6$.

\subsection{Growing boundary layer}

From the results in $\S 3$, we observed the following two crucial trends under certain flow conditions: (a) a steady state is achieved in the immediate vicinity of $\Theta=0$, but, $(b)$, in an outer zone of the boundary layer there is continuing growth of the boundary-layer thickness with time. Therefore, we look for a double structure to the boundary layer.

Consider first the outer region, for which we introduce again the variable (2.24), $\eta=\Theta t^{-1 / 2}=O(1)$, where in general

$$
\begin{gathered}
\hat{W}=\bar{W}(\eta)+\ldots, \\
\hat{V}=t^{-1 / 2} \bar{V}(\eta)+\ldots, \\
B^{*}=\bar{B}(\eta)+\ldots
\end{gathered}
$$

The resulting system is then

$$
\begin{gathered}
\bar{W}^{2}=\bar{B}, \\
\bar{W}_{\eta \eta}=-\frac{1}{2} \eta \bar{W}_{\eta}-\bar{V}_{\eta} \bar{W}+\bar{V} \bar{W}_{\eta}, \\
\frac{1}{\sigma} \bar{B}_{\eta \eta}=-\frac{1}{2} \eta \bar{B}_{\eta}-\frac{1}{2} \bar{V}_{\eta} \bar{B}+\bar{V} \bar{B}_{\eta}+\frac{1}{2} S^{*} \bar{V}_{\eta} .
\end{gathered}
$$

The appropriate boundary conditions are

$$
\bar{W}=1, \quad \bar{V}=\bar{V}_{0}=\text { constant } \quad \text { on } \quad \eta=0,
$$


together with the conditions

$$
\bar{U} \rightarrow 0, \quad \bar{W} \rightarrow \hat{W}_{e}, \quad \bar{B} \rightarrow \hat{W}_{e}^{2} \quad \text { for } \quad \eta \rightarrow-\infty .
$$

On account of (4.4) and (4.7), we must have $\bar{B}(\eta=0)=1$, so that the fourth condition in (2.18) is not satisfied. Hence, there is a need for an inner layer, described by $\Theta=O(1)$; on this scale, the solution develops in the following manner:

$$
\begin{gathered}
B^{*}=B_{0}+t^{-1 / 2} B_{1}(\Theta)+\ldots, \\
\hat{W}=1+t^{-1 / 2} W_{1}(\Theta)+\ldots, \\
\hat{U}=t^{-1 / 2} U_{1}(\Theta)+\ldots, \\
\hat{V}=t^{-1 / 2} V_{1}(\Theta)+\ldots,
\end{gathered}
$$

where

$$
B_{0}=1
$$

The leading-order governing equations are then

$$
\begin{gathered}
-2 W_{1}=U_{1 \Theta \Theta}-B_{1}, \\
2 U_{1}=W_{1 \Theta \Theta}, \\
-S^{*} U_{1}+B_{0} U_{1}=\frac{1}{\sigma} B_{1 \Theta \Theta}, \\
2 U_{1}+V_{1 \Theta}=0,
\end{gathered}
$$

together with

$$
U_{1}(0)=V_{1}(0)=W_{1}(0)=B_{\Theta}(0)=0,
$$

and, to match with the outer layer,

$$
U_{1} \rightarrow 0, \quad W_{1} \sim \bar{W}_{\eta}(0) \Theta, \quad B_{1} \sim \bar{B}_{\eta}(0) \Theta \quad \text { as } \quad \Theta \rightarrow-\infty .
$$

We note that $2 \bar{W}_{\eta}(0)=\bar{B}_{\eta}(0)$. Equations (4.14)-(4.19) may now be solved in a straightforward manner to yield

$$
\begin{gathered}
U_{1}=\frac{2 \lambda \bar{B}_{\eta}(0)}{\sigma\left(B_{0}-S^{*}\right)} \sin (\lambda \Theta) \mathrm{e}^{\lambda \Theta}, \\
B_{1}=\bar{B}_{\eta}(0)\left\{\Theta-\frac{\cos (\lambda \Theta) \mathrm{e}^{\lambda \Theta}}{\lambda}+\frac{4}{\lambda \sigma\left(B_{0}-S^{*}\right)}\right\}, \\
V_{1}=\frac{2 \bar{B}_{\eta}(0)}{\sigma\left(B_{0}-S^{*}\right)}\left\{\mathrm{e}^{\lambda \Theta} \cos (\lambda \Theta)-\mathrm{e}^{\lambda \Theta} \sin (\lambda \Theta)-1\right\},
\end{gathered}
$$

where $\lambda^{4}=1-\frac{1}{4} \sigma\left(B_{0}-S^{*}\right) ; W_{1}$ then follows from (4.14).

These inner-layer solutions, in particular (4.22), enable us to close the outer problem, (4.4)-(4.8). We take the limit of (4.22) as $\Theta \rightarrow-\infty$, to give

$$
\bar{V}_{0}=-\frac{2 \bar{B}_{\eta}(0)}{\sigma\left(B_{0}-S^{*}\right)}
$$

which, by linking $\bar{V}(0)$ to $\bar{B}_{\eta}(0)$ is sufficient to uniquely determine the solution. The $\eta=O(1)$ computation was carried out using a fourth-order shooting method, coupled with Newton iteration. Figure 7 shows a comparison of the asymptotic value of $\bar{B}(\eta)$ with the scaled fully numerical solution, $B^{*}\left(\Theta t^{-1 / 2}\right)$, for the specific case $\hat{W}_{e}=1.5$, 


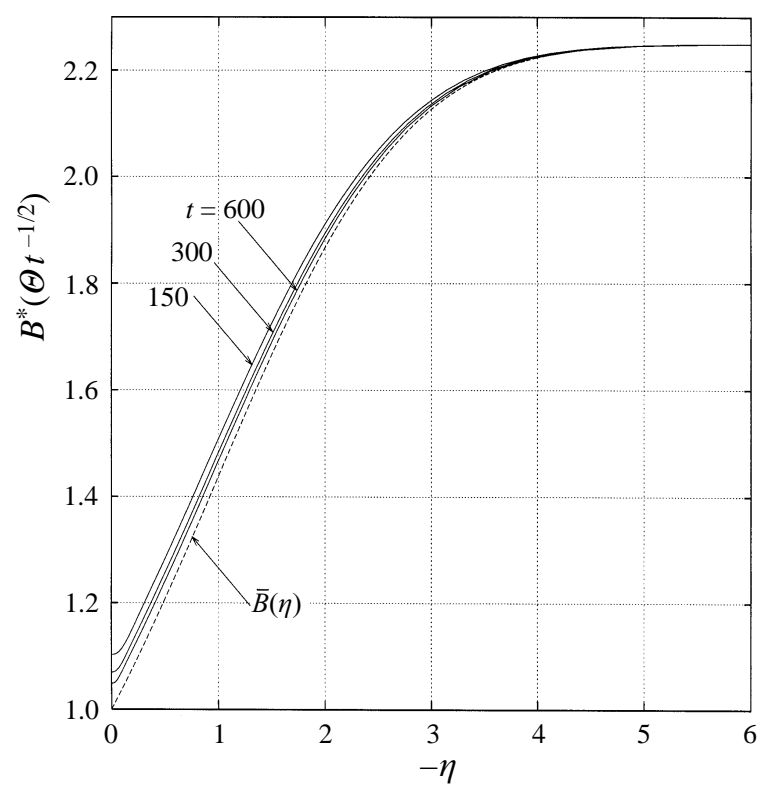

FIGURE 7. Comparison of $B^{*}\left(\Theta t^{1 / 2}\right)$ with $\bar{B}(\eta): \hat{W}_{e}=1.5, \sigma=1, S^{*}=2.75$.

$\sigma=1, S^{*}=2.75$ (at $t=150,300,600$ ). We clearly see the fully numerical solution approaching the asymptotic state, in a satisfactory manner. It should be noted that this solution is qualitatively similar to that found by MacCready \& Rhines (1991).

\subsection{Nature of the finite-time breakdown}

The nature of the finite-time breakdown, found to occur in some regions of $\left(S^{*}, \hat{W}_{e}\right)$ space, is of particular interest since it would play a very significant role in the global spin-up or spin-down of the fluid in such a container. Specifically a sensible balancing of terms within the governing equations leads to a new inner-scale dependency, given by $\tilde{\eta}=\Theta /\left(t_{0}-t\right)^{1 / 2}$ as $t \rightarrow t_{0}$, the time at breakdown. This balancing also suggests the following solution development on the $\tilde{\eta}=O(1)$ scale:

$$
\begin{aligned}
& \hat{U}=\left(t_{0}-t\right)^{-1} \hat{U}_{1}(\tilde{\eta})+\ldots, \\
& B^{*}=\left(t_{0}-t\right)^{-2} \hat{B}_{1}(\tilde{\eta})+\ldots, \\
& \hat{W}=\left(t_{0}-t\right)^{-1} \hat{W}_{1}(\tilde{\eta})+\ldots, \\
& \hat{V}=\left(t_{0}-t\right)^{-1 / 2} \hat{V}_{1}(\tilde{\eta})+\ldots
\end{aligned}
$$

The resulting system is then

$$
\begin{gathered}
\hat{U}_{1}+\frac{1}{2} \tilde{\eta} \hat{U}_{1 \tilde{\eta}}+\hat{U}_{1}^{2}+\hat{V}_{1} \hat{U}_{1 \tilde{\eta}}-\hat{W}_{1}^{2}=\hat{U}_{1 \tilde{\eta} \tilde{\eta}}-\hat{B}_{1}, \\
\hat{W}_{1}+\frac{1}{2} \tilde{\eta} \hat{W}_{1 \tilde{\eta}}+2 \hat{U}_{1} \hat{W}_{1}+\hat{V}_{1} \hat{W}_{1 \tilde{\eta}}=\hat{W}_{1 \tilde{\eta} \tilde{\eta}}, \\
2 \hat{B}_{1}+\frac{1}{2} \tilde{\eta} \hat{B}_{1 \tilde{\eta}}+\hat{V}_{1} \hat{B}_{1 \tilde{\eta}}+\hat{U}_{1} \hat{B}_{1}=\frac{1}{\sigma} \hat{B}_{1 \tilde{\eta} \tilde{\eta}}, \\
2 \hat{U}_{1}+\hat{V}_{1 \tilde{\eta}}=0,
\end{gathered}
$$

with boundary conditions

$$
\hat{W}_{1}=1, \quad \hat{U}_{1}=\hat{V}_{1}=\hat{B}_{1 \tilde{\eta}}=0 \quad \text { on } \quad \tilde{\eta}=0 .
$$


As $\tilde{\eta} \rightarrow-\infty$, the only meaningful conditions are that

$$
\hat{U}_{1}=O\left(\tilde{\eta}^{-2}\right), \quad \hat{W}_{1}=O\left(\tilde{\eta}^{-2}\right), \quad \hat{B}_{1}=O\left(\tilde{\eta}^{-4}\right), \quad \hat{V}_{1}=O(1) .
$$

There is a still thinner inner scale, given by $\eta=O\left(t_{0}-t\right)$, where the leadingorder $\hat{W}$ term becomes $O(1)$, in order to satisfy the no-slip condition on the conical surface; however, this region is entirely passive. There is additionally an outer region, $\Theta=O(1)$, in which the solution develops in the following manner:

$$
\begin{gathered}
\hat{U}=U_{1}^{*}(\Theta)+\left(t_{0}-t\right)^{1 / 2} U_{2}^{*}(\Theta)+\ldots, \\
\hat{V}=\left(t_{0}-t\right)^{-1 / 2} V_{0}^{*}+V_{1}^{*}(\Theta)+\left(t_{0}-t\right)^{1 / 2} V_{2}^{*}(\Theta)+\ldots, \\
\hat{W}=W_{1}^{*}(\Theta)+\left(t_{0}-t\right)^{1 / 2} W_{2}^{*}(\Theta)+\ldots, \\
B^{*}=B_{1}^{*}(\Theta)+\left(t_{0}-t\right)^{1 / 2} B_{2}^{*}(\Theta)+\ldots,
\end{gathered}
$$

where

$$
V_{0}^{*}=\hat{V}(\tilde{\eta} \rightarrow-\infty)=\text { constant. }
$$

We can determine nothing about the nature of $\left(U_{1}^{*}, V_{1}^{*}, W_{1}^{*}, B_{1}^{*}\right)$, which must reflect the history of the problem, and may therefore only be determined by the full numerical solution. The $O\left(\left(t_{0}-t\right)^{1 / 2}\right)$ terms are determined to be

$$
\begin{aligned}
U_{2}^{*} & =2 V_{0}^{*} U_{1 \Theta}^{*}, \\
W_{2}^{*} & =2 V_{0}^{*} W_{1 \Theta}^{*}, \\
B_{2}^{*} & =2 V_{0}^{*} B_{1 \Theta}^{*} .
\end{aligned}
$$

The key problem is then the solution of (4.28)-(4.33), which is in fact a nonlinear eigenvalue problem. Solution of this system was achieved by means of a finite-difference algorithm, including Newton iteration to force the correct boundary conditions on $\tilde{\eta}=0$. At first it was difficult to obtain non-trivial solutions of this system, but ultimately a (scaled) solution from the fully numerical approach was used as an 'initial guess', and the algorithm then worked well. Figures $8(a)$ and $8(b)$ show a comparison of our asymptotic profile for $\hat{B}_{1}(\tilde{\eta})$ and $\hat{V}_{1}(\tilde{\eta})$ respectively, with scaled fully numerical solutions just prior to breakdown (for the case $\sigma=1, \hat{W}_{e}=2.0, S^{*}=0.5$ ). There is good agreement between the two sets of results, adding credence to the asymptotic approach.

\section{An overview of parameter space}

There must clearly be boundaries in an $\left(S^{*}, \hat{W}_{e}\right)$-space that separate the three classes of ultimate behaviour, i.e. (i) the steady state, (ii) the finite-time singularity and (iii) the growing boundary-layer scenario.

It is easy, computationally, to differentiate case (ii) from the others, but the distinction between (i) and (iii) is more difficult to discern. However, a series of numerical investigations categorically revealed that in $\left(S^{*}, \hat{W}_{e}\right)$-space the boundary between (i) and (ii) is $\hat{W}_{e}=1$ for $S^{*} \leqslant 1$, whilst the boundary between (ii) and (iii) is the curve $S^{*}=\hat{W}_{e}^{2}$ for $\hat{W}_{e}>1$. In $\hat{W}_{e}<1$, the boundary between (i) and (iii) is more complicated, and is found to be dependent on the value of $\sigma$. We denote the functional dependence of this latter boundary by $S_{\text {crit }}^{*}\left(\hat{W}_{e}\right)$.

In order to facilitate the determination of the boundary between the flow regimes (i) and (iii), a fully steady computer code (based on (2.7), (2.9), (2.16)-(2.19) with 

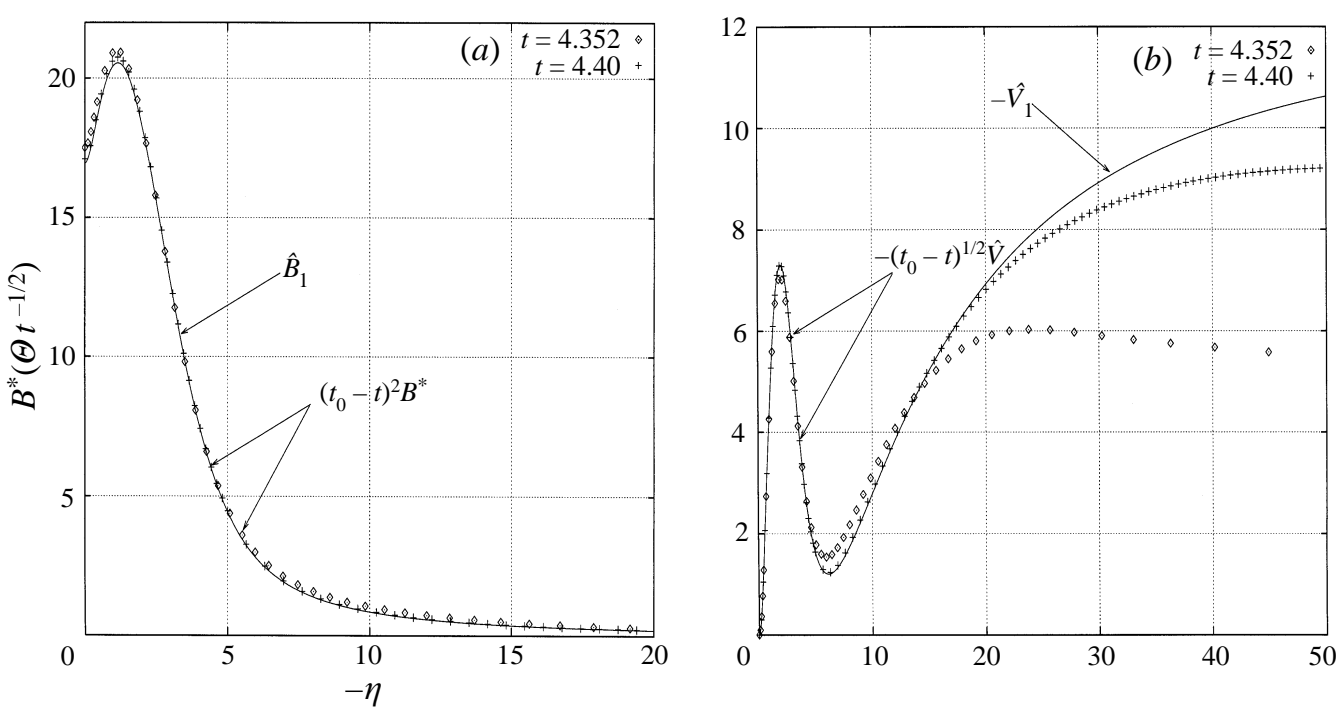

FIGURE 8. Comparison of $(a) \hat{B}_{1}(\tilde{\eta})$ with $\left(t_{0}-t\right)^{2} B^{*}\left(\Theta /\left(t_{0}-t\right)^{1 / 2}\right)$ and $(b) \hat{V}_{1}(\tilde{\eta})$ with $\left(t_{0}-t\right)^{1 / 2} \hat{V}\left(\Theta /\left(t_{0}-t\right)^{1 / 2}\right)$.

the acceleration terms omitted) was used, employing a second-order-accurate, finitedifference scheme in $\Theta$, with Newton iteration to treat the nonlinearity of the problem. The results of this numerical approach prompted a further asymptotic description in the region of $S_{c r i t}^{*}$. We can introduce an expansion analogous to that of the growing boundary-layer analysis (§4.1); here, a perturbation away from $S_{c r i t}^{*}$,

$$
S^{*}=S_{c r i t}^{*}+\delta,
$$

leads to the following expansions:

$$
\begin{aligned}
\hat{U} & =\delta U_{1}(\Theta)+\ldots, \\
\hat{V} & =\delta V_{1}(\Theta)+\ldots, \\
\hat{W} & =1+\delta W_{1}(\Theta)+\ldots, \\
B^{*} & =1+\delta B_{1}(\Theta)+\ldots,
\end{aligned}
$$

in an $O(1)$ layer near the boundary. In this region we obtain a linear set of equations in the form of (4.14)-(4.17) but with $\left\{B_{0}, S^{*}\right\}$ replaced by $\left\{1, S_{c r i t}^{*}\right\}$ and the boundary conditions (4.18). Solution of this inner problem shows that, as $\Theta \rightarrow-\infty$,

$$
U_{1} \rightarrow 0, \quad V_{1} \rightarrow \gamma, \quad W_{1} \sim \gamma \Theta\left[\lambda^{4}-1\right],
$$

where $\gamma$ is a constant and $\lambda$ is defined by

$$
\lambda^{4}=\frac{1}{4}\left[4-\sigma\left(1-S_{\text {crit }}^{*}\right)\right] .
$$

The results for $|\Theta| \gg 1$, coupled with (5.2)-(5.5) suggest an outer layer,

$$
\tilde{\Theta}=\delta \Theta=O(1),
$$

in which the appropriate expansions are

$$
\begin{aligned}
& \hat{U}=\delta^{2} \tilde{U}_{1}(\tilde{\Theta})+\ldots, \\
& \hat{V}=\delta \tilde{V}_{1}(\tilde{\Theta})+\ldots,
\end{aligned}
$$




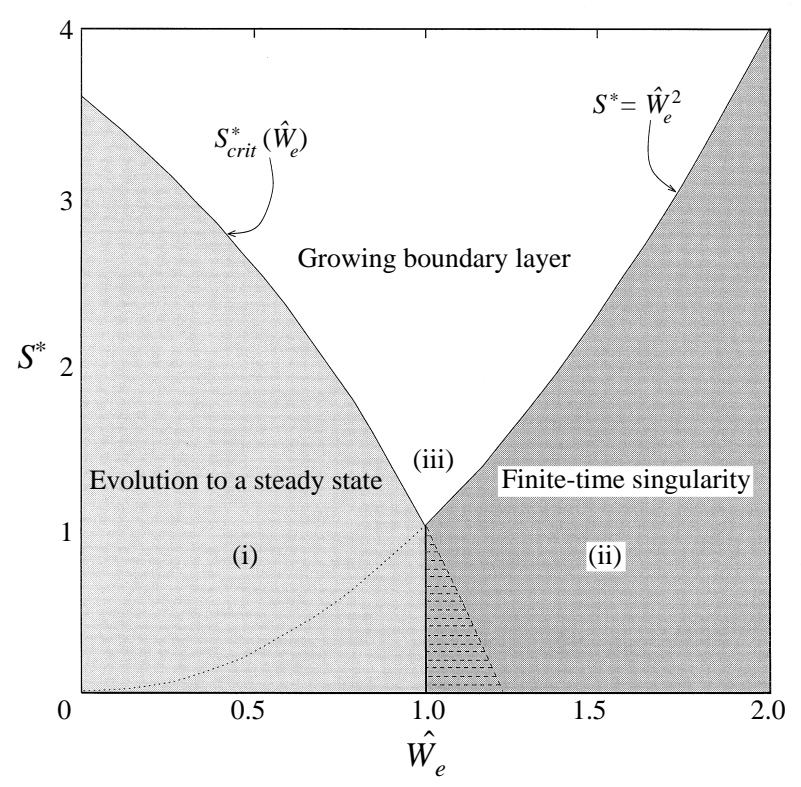

FIGURE 9. Different flow regimes, $\sigma=1$.

$$
\begin{aligned}
\hat{W} & =\tilde{W}_{1}(\tilde{\Theta})+\ldots, \\
B^{*} & =\tilde{B}_{1}(\tilde{\Theta})+\ldots
\end{aligned}
$$

The governing equations in this region are of the form (4.4)-(4.6) but without terms of the form $\eta \partial / \partial \eta$, and with $\left\{\bar{V}, \bar{W}, \bar{B}, S^{*}\right\}$ replaced by $\left\{\tilde{V}_{1}, \tilde{W}_{1}, \tilde{B}_{1}, S_{c r i t}^{*}\right\}$. The resulting system can be reduced to a third-order problem, by eliminating $U_{1}$ and $\tilde{B}_{1}$, with boundary conditions $\tilde{W}_{1}(0)=1, \tilde{W}_{1}^{\prime}(0)=\gamma\left[\lambda^{4}-1\right], \tilde{V}_{1}(0)=\gamma$, where $\gamma$ can be set arbitrarily to unity, corresponding to the redefinition

$$
\tilde{V}_{1 \text { new }}=\frac{\tilde{V}_{1}}{\gamma}, \quad \tilde{\Theta}_{\text {new }}=\gamma \tilde{\Theta}=\gamma \delta \Theta .
$$

This outer problem can now be solved with a simple shooting method to determine $S_{c r i t}^{*}\left(\hat{W}_{e}\right)$. Matching the above asymptotics to the final states obtained from the unsteady problem, in the steady-state region (i), determines $\gamma$ to be negative and, for an exponentially decaying solution to the outer problem to exist, forces $\delta<0$ (so that $S^{*}<S_{\text {crit }}^{*}$ and $0 \geqslant \tilde{\Theta}>-\infty$ is the appropriate solution domain). As $S^{*}$ increases, the stable, steady solutions cannot be continued above $S^{*}=S_{\text {crit }}^{*}$, because $0 \leqslant \tilde{\Theta}<\infty$ becomes the appropriate domain. The unsteady problem then evolves to a growing boundary-layer state with a similar double-layer structure.

The results of our computations are displayed in the regime diagram, figure 9, which is computed for the particular case $\sigma=1$. Steady-state solutions exist in the lightly shaded region, $S^{*}<S_{c r i t}^{*}\left(\hat{W}_{e}\right)$ for $\hat{W}_{e}<\hat{W}_{c r i t}$, where $\hat{W}_{c r i t}>1$ is a constant found to be dependent on $\sigma$. The other shaded zone, given by $S^{*}<\hat{W}_{e}^{2}$ and $\hat{W}_{e}>1$, corresponds to parameter sets for which a finite-time singularity occurs, case (ii). The remainder of the diagram, unshaded, corresponds to situation (iii), which is the growing boundary-layer region. We find that in some regions of parameter space where steady-state solutions exist, growing-boundary-layer solutions 


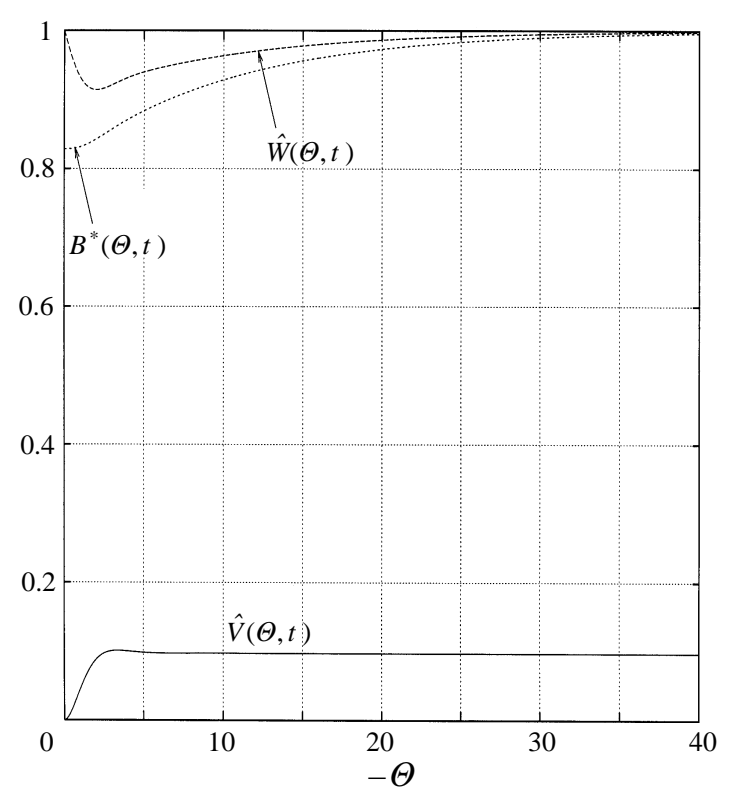

FIGURE 10. Non-trivial profiles, $S^{*}=0.5, \sigma=1, \hat{W}_{e}=1$.

are simultaneously possible, but these solutions were never attained by our unsteady initial-value computations.

Intriguingly, for $\hat{W}_{e}=1, S^{*}<1$, although a trivial solution exists in this regime $\left(\hat{W}=1, B^{*}=1, \hat{U}=\hat{V}=0\right)$, this solution was generally not attained by the steady algorithm; instead, profiles of the type shown in figure 10 (for the particular case $\left.S^{*}=\frac{1}{2}, \sigma=1\right)$ were obtained. Additionally, it was found that steady solutions for $S^{*}<1$ were possible, for $1<\hat{W}_{e}<\hat{W}_{\text {crit }}$, even though the unsteady computations generally led to finite-time singularities in this regime. One note of caution worth mentioning is that in the above discussion we have tacitly assumed that the upper boundary of the steady-state solution $\left(S_{\text {crit }}^{*}\left(\hat{W}_{e}\right)\right.$, for $\hat{W}_{e}<1$ in figure 9) corresponds to the lower boundary of the unsteady boundary-layer solution. We believe this to be the case.

The point $\hat{W}_{e}=S^{*}=1$ is of some interest, representing what appears to be a triple point in the neighbourhood of which all three classes of flow behaviour can be observed.

\section{Large values of $\sigma$}

As noted in our introduction, in a number of practical situations the Prandtl (or Schmidt) number is large: in particular, for laboratory experiments involving a solution of salt in water, $\sigma \approx 1000$. In this section we consider the limit of large values of $\sigma$. In figure 11 we show the temporal development for the case $S^{*}=\frac{1}{2}$, $\hat{W}_{e}=0$ for $\sigma=1,25,200,1000$. This is a spin-up from rest case, for which, as previously discussed, the long-time solution is steady for $\sigma=O(1)$. Here too, all $\sigma$ cases approach a steady state.

We now examine the solutions of the equations by asymptotic means, for $\sigma \gg 1$. Figure 11 suggests that $B^{*}(\Theta=0)$ grows as $\sigma$ increases, whilst $\hat{W}_{\Theta}(\Theta=0)$ becomes 


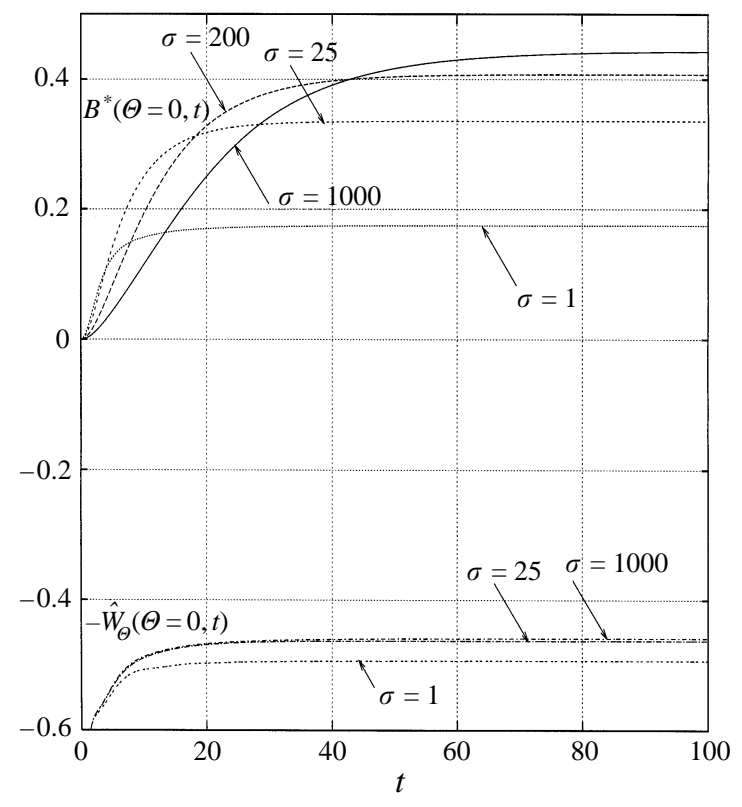

FIGURE 11. Temporal development for $S^{*}=0.5, \hat{W}_{e}=0$, with $\sigma$ as indicated.

independent of $\sigma$ in this limit. Guided by these observations, we write

$$
\begin{gathered}
B^{*}=\hat{B}^{*}(\hat{\theta}, \hat{t})+\ldots, \\
\hat{U}=\sigma^{-1 / 3} \kappa \hat{\theta}+\ldots, \\
\hat{V}=-\sigma^{-2 / 3} \kappa \hat{\theta}^{2}+\ldots, \\
\hat{W}=1+\sigma^{-1 / 3} \kappa_{1} \hat{\theta}+\ldots, \\
\hat{\theta}=\sigma^{1 / 3} \Theta, \\
\hat{t}=\sigma^{-1 / 3} t,
\end{gathered}
$$

where $\kappa$ and $\kappa_{1}$ are constants. Substitution of this asymptotic expansion into (2.7), (2.9), (2.16) and (2.17) leads to the following equation for $\hat{B}^{*}$ :

$$
\frac{\partial \hat{B}^{*}}{\partial \hat{t}}-\kappa \hat{\theta}^{2} \frac{\partial \hat{B}^{*}}{\partial \hat{\theta}}-S^{*} \kappa \hat{\theta}+\kappa \hat{\theta} \hat{B}^{*}=\frac{\partial^{2} \hat{B}^{*}}{\partial \hat{\theta}^{2}}
$$

with $\hat{B}^{*} \rightarrow S^{*}$ as $\hat{\theta} \rightarrow-\infty$ and $\left.\hat{B}_{\hat{\theta}}^{*}\right|_{\hat{\theta}=0}=0$. What we have is typical of high-Prandtlnumber boundary layers: a thin buoyancy layer, in which the velocity components are Taylor-series expansions from a thicker velocity boundary layer, which involves a velocity variation with only weak changes in $B^{*}$.

In the outer, velocity boundary layer, $\Theta=O(1)$ and all quantities are $O(1)$, and are in fact determined through the steady equations, with conditions $\hat{U}=\hat{V}=0, \hat{W}=$ $1, B^{*}=S^{*}$ on $\Theta=0$ and with edge conditions given by (2.19). The quantities $\kappa$ and $\kappa_{1}$ which appear in the expressions (6.2)-(6.4) are then determined from the solution in this steady outer layer.

The above analysis is confirmed by figure 11, which reveals that the flow development for $\hat{W}_{\Theta}(\Theta=0)$ is almost invariant with $\sigma$, although $B^{*}(\Theta=0)$ grows markedly with $\sigma$. Additionally, steady-state wall values of $B^{*}$ take considerably 
longer to achieve than do the corresponding values of $\hat{W}_{\Theta}(\Theta=0)$, consistent with the asymptotic description above.

Overall we found that the types of flow behaviour for $\sigma=1$ are mirrored in the $\sigma \gg 1$ results; in particular, the $\left(S^{*}, \hat{W}_{e}\right)$ parameter space is divided into zones similar to those shown in figure 9 for $\sigma=1$.

\section{Linearized solution}

Here, we return to the problem studied by MacCready \& Rhines (1991), namely the linearised version of (1.1)-(1.7) for $\omega$ only slightly different from unity. The approach to the linearized problem in this section is to use asymptotic methods on the exact Laplace transform, in order to obtain a complete picture of the long-time evolution of the boundary layer. The reasons for this approach are twofold: first, we will fill in some missing elements in the prior study (MacCready \& Rhines 1991), in order to be sure that the flow picture is complete; second, to provide some insight into solutions in the immediate neighbourhood of $\hat{W}_{e}=1$, which we know from $\S 5$ is an area of interest within the parameter space of figure 9 . It is convenient in this case to return to a frame of reference rotating rigidly at angular speed $\Omega$, so now the velocity components $(u, v,-w)$ are perturbations of $\left(v_{r}, v_{\theta}, v_{\phi}\right)$ away from the rigid rotation, and the notation is consistent with that of MacCready \& Rhines (1991). The quantity $z$ is a boundary-layer coordinate given by $-r \theta$, in the conical case. Since continuity now de-couples from the others, equations (1.2)-(1.5) become

$$
\begin{gathered}
\frac{\partial u}{\partial t}-2 \cos \alpha\left(w-w_{e}\right)=\frac{\partial^{2} u}{\partial z^{2}}-\sin \alpha\left(B-B_{e}\right), \\
\frac{\partial w}{\partial t}+2 \cos \alpha u=\frac{\partial^{2} w}{\partial z^{2}}, \\
\frac{\partial B}{\partial t}-S \sin \alpha u=\frac{1}{\sigma} \frac{\partial^{2} B}{\partial z^{2}},
\end{gathered}
$$

where we have replaced the radial pressure gradient with terms involving edge values of $B, w$ in the usual way. We assume that the initial fluid state is given by $u=0$, $w \equiv w_{e}, B \equiv B_{e}$, and at $t=0$ the wall is impulsively changed to a speed $w=w_{b}$, and simultaneously the buoyancy-induced 'slope current' starts to develop, due to imposing (7.3). The initial conditions are not very important, since we are principally interested in the long-time solutions of these equations, which will be applicable to constructing solutions to a global spin-up problem. The boundary conditions are stated a few lines below, in Laplace-transformed form.

It is convenient to make a series of variable changes in order to simplify the algebra in the subsequent analysis. Hence, we redefine the boundary-layer coordinate by $z=(2 \cos \alpha)^{-1 / 2} \zeta$ and the time variable by $t=(2 \cos \alpha)^{-1} T$. Let the buoyancy also be rewritten as $B=2 \cot \alpha \hat{B}$. This transformation generates a set of partial differential equations in $(\zeta, T)$ identical to (7.1)-(7.3), but with all factors of 2 removed, all trigonometric functions set to 1 and a new Burger number, $\tilde{S} \equiv S \tan ^{2} \alpha / 4$, replacing the $S$ in (7.3). This ' $S$ ' is identical to the Burger number used by MacCready \& Rhines (1991), and our azimuthal velocity component $w$ is $v$ in their paper.

To proceed to a time-dependent solution of these transformed equations, we write

$$
u=\tilde{u}, \quad v=\tilde{v}, \quad w=w_{e}+\tilde{w}, \quad \hat{B}=\hat{B}_{e}+\tilde{B} .
$$

Although it is possible to solve these equations in an ad hoc fashion, it seems best, 
in order to not miss any details, to use a Laplace transform technique in time, $T$. Letting $\mathscr{L}$ denote the usual Laplace transformation operator, it is easy to verify that the solution of the transformed system can be written as

$$
\begin{gathered}
\mathscr{L}\{\tilde{u}\}=C_{1} \mathrm{e}^{-\lambda_{1} \zeta}+C_{2} \mathrm{e}^{-\lambda_{2} \zeta}+C_{3} \mathrm{e}^{-\lambda_{3} \zeta}, \\
\mathscr{L}\{\tilde{w}\}=-\frac{C_{1}}{s-\lambda_{1}^{2}} \mathrm{e}^{-\lambda_{1} \zeta}-\frac{C_{2}}{s-\lambda_{2}^{2}} \mathrm{e}^{-\lambda_{2} \zeta}-\frac{C_{3}}{s-\lambda_{3}^{2}} \mathrm{e}^{-\lambda_{3} \zeta}, \\
\mathscr{L}\{\tilde{B}\}=\frac{\tilde{S} C_{1}}{s-\lambda_{1}^{2} / \sigma} \mathrm{e}^{-\lambda_{1} \zeta}+\frac{\tilde{S} C_{2}}{s-\lambda_{2}^{2} / \sigma} \mathrm{e}^{-\lambda_{2} \zeta}+\frac{\tilde{S} C_{3}}{s-\lambda_{3}^{2} / \sigma} \mathrm{e}^{-\lambda_{3} \zeta},
\end{gathered}
$$

where $s$ is the Laplace-domain complex variable. The quantities $\left\{\lambda_{i}\right\}$ are the three solutions, with positive real part, of the sixth-degree polynomial

$$
\left(s-\lambda^{2}\right)^{2}\left(s-\frac{\lambda^{2}}{\sigma}\right)+s(1+\tilde{S})-\lambda^{2}\left(\tilde{S}+\frac{1}{\sigma}\right)=0 .
$$

The boundary conditions stated in the general case (see (1.6) and (1.7)) become

$$
\left.\begin{array}{c}
\mathscr{L}\{\tilde{u}\}=0, \quad \mathscr{L}\{\tilde{w}\}=-\frac{w_{e}-w_{b}}{s}, \\
\frac{\partial \mathscr{L}\{\tilde{B}\}}{\partial \zeta}=\frac{A \tilde{S}}{s} \quad \text { at } \quad \zeta=0, \\
A \equiv(2 \cos \alpha E)^{1 / 2} \cot \alpha .
\end{array}\right\}
$$

Application of these conditions gives the three simultaneous equations for the quantities $\left\{C_{i}\right\}$; one simply relates $C_{3}=-C_{1}-C_{2}$; the others may be put in a more convenient form by writing $K_{i} \equiv C_{i}\left(\lambda_{i}-\lambda_{3}\right)$, and are

$$
\left(\begin{array}{cc}
\frac{\lambda_{1}+\lambda_{3}}{s-\lambda_{1}^{2}} & \frac{\lambda_{2}+\lambda_{3}}{s-\lambda_{2}^{2}} \\
s+\lambda_{1} \lambda_{3} / \sigma & \frac{s+\lambda_{2} \lambda_{3} / \sigma}{s-\lambda_{2}^{2} / \sigma}
\end{array}\right) \boldsymbol{K}=\boldsymbol{Q},
$$

where $\boldsymbol{K}=\left(K_{1}, K_{2}\right)^{\mathbf{T}}$, and the vector $\boldsymbol{Q}$ is given by

$$
\boldsymbol{Q}=\frac{1}{s}\left(\begin{array}{c}
\left(s-\lambda_{3}^{2}\right)\left(w_{e}-w_{b}\right) \\
-\left(s-\lambda_{3}^{2} / \sigma\right) A
\end{array}\right)
$$

Equation (7.10) may be solved by Cramer's rule; the determinant of the matrix in (7.10) is

$$
\left.\begin{array}{c}
\Delta=\frac{N\left(\lambda_{2}-\lambda_{1}\right)}{\left(s-\lambda_{1}^{2}\right)\left(s-\lambda_{2}^{2}\right)\left(s-\lambda_{1}^{2} / \sigma\right)\left(s-\lambda_{2}^{2} / \sigma\right)}, \\
N=\frac{\lambda_{3}^{2}}{\sigma}\left[s^{2}-s\left(\lambda_{1}^{2}+\lambda_{2}^{2}\right)+\lambda_{1} \lambda_{2}\left(\frac{\lambda_{1} \lambda_{2}}{\sigma}-s\left(1-\frac{1}{\sigma}\right)\right)\right] \\
-\lambda_{3} s\left(1-\frac{1}{\sigma}\right)\left(\lambda_{1}+\lambda_{2}\right)+\frac{s^{2}}{\sigma}\left(\lambda_{1}^{2}+\lambda_{2}^{2}+\lambda_{1} \lambda_{2}\right)-s^{3}-\frac{s}{\sigma} \lambda_{1}^{2} \lambda_{2}^{2}-s^{2} \lambda_{1} \lambda_{2} .
\end{array}\right\}
$$

Clearly, exact inversion of these transforms is not possible, but it is not necessary since, in the context of spin-up applications, we are principally interested in large times, $T \gg 1$. So, we may examine the functions $\left\{C_{i}, \lambda_{i}\right\}$ to determine the long-time behaviour of solutions to (7.1)-(7.3). In particular, any singularities of the transforms 
(7.5)-(7.7) in $\operatorname{Re}(s)>0$ will lead to secular growth in time. It appears, from a careful examination of the roots to (7.8), that there are no singularities in the right half-plane, at least for $\tilde{S}>0$, which is assumed here. In $\S 7.3$, we will show a connection between these solutions and certain special solutions of the nonlinear problems of $\S \S 3-5$; it will be seen that $\tilde{S}$ may be negative, and therefore the question of root location must be revisited. For now however, for the purposes of comparison with the previous linearized analyses (Thorpe 1987; MacCready \& Rhines 1991), we shall take $\tilde{S}>0$ and for this case there are no roots of $(7.8)$ in $\operatorname{Re}(s)>0$.

Hence, the behaviour of the transforms near the imaginary axis in the s-plane will determine the long-time behaviour. The general inversion contour in the $s$-plane may be deformed about any such singularities, with the legs of the paths going far into the left half of the plane. Obviously the origin itself is of crucial importance to the long-time behaviour, but on the other hand, depending on their strength, some singularities on $\operatorname{Re}(s)=0$ may produce time-periodic components of the long-time solution.

\subsection{Inverse structure near $s=0$}

To consider first the behaviour of the transforms of $\tilde{u}, \tilde{w}, \tilde{B}$ in the neighbourhood of the origin in the complex s-plane, we need the behaviour of the roots to (7.8) as $s \rightarrow 0$,

$$
\begin{gathered}
\lambda_{1,2}=(1 \pm \mathrm{i}) \gamma+O(s), \quad \gamma \equiv((1+\tilde{S} \sigma) / 2)^{1 / 4}, \\
\lambda_{3} \sim \beta s^{1 / 2}+O\left(s^{3 / 2}(\sigma-1)\right), \quad \beta \equiv\left(\frac{1+\tilde{S}}{1 / \sigma+\tilde{S}}\right)^{1 / 2} .
\end{gathered}
$$

With these approximations for $\left\{\lambda_{1}, \lambda_{2}, \lambda_{3}\right\}$, the determinant $\Delta$ may be found to be

$$
\Delta \sim-\frac{\mathrm{i} \sigma s}{2 \gamma^{3}}\left[\left(\frac{\beta^{2}}{\sigma}-1\right)-\frac{\beta s^{1 / 2}(\sigma-1)}{2 \gamma^{3}}+O(s)\right] .
$$

Then, (7.10) may be solved to give the behaviour of $\left\{C_{1}, C_{2}, C_{3}\right\}$ in the neighbourhood of $s=0$, namely

$$
\left.\begin{array}{l}
C_{1} \sim-C_{2} \sim-\frac{\mathrm{i} \gamma}{s \sigma}\left[-A+\beta\left(w_{e}-w_{b}\right) s^{1 / 2} \frac{1-\beta^{2}}{1-\beta^{2} / \sigma}+O(s)\right], \\
C_{3} \sim\left(1-\beta^{2}\right)\left(w_{e}-w_{b}+\frac{A}{\gamma \sigma}\right)+O\left(s^{1 / 2}\right) .
\end{array}\right\}
$$

These expressions for $\left\{C_{1}, C_{2}, C_{3}\right\}$ are valid only in the immediate neighbourhood of $s=0$, failing in most of $\operatorname{Re}(s)<0$. Differences between these transforms and the exact expressions will lead to vanishingly small effects as $T \rightarrow \infty$, provided that no other poles or branch points lie at or near $\operatorname{Re}(s)=0$. We return to this question below, but for now, based on (7.13), (7.14) and (7.16), we invert (7.5)-(7.7) to obtain

$$
\begin{gathered}
\tilde{u} \sim-\left[\frac{A \gamma}{\sigma}+\frac{2}{(\pi T)^{1 / 2}} \frac{\gamma \beta}{\sigma \tilde{S}}\left(w_{e}-w_{b}\right)\right] \sin (\gamma \zeta) \mathrm{e}^{-\gamma \zeta}+K\left(1-\beta^{2}\right) \frac{\zeta \beta}{2\left(\pi T^{3}\right)^{1 / 2}} \mathrm{e}^{-\zeta^{2} \beta^{2} / 4 T}, \\
\tilde{w} \sim\left[\frac{A}{\gamma \sigma}+\frac{1}{(\pi T)^{1 / 2}} \frac{\gamma \beta}{\sigma \tilde{S}}\left(w_{e}-w_{b}\right)\right] \cos (\gamma \zeta) \mathrm{e}^{-\gamma \zeta}-K \operatorname{erfc}\left(\frac{\beta \zeta}{2 T^{1 / 2}}\right),
\end{gathered}
$$




$$
\tilde{B} \sim-\left[\frac{A}{\gamma}+\frac{1}{(\pi T)^{1 / 2}} \frac{4 \beta}{\gamma \tan ^{2} \alpha}\left(w_{e}-w_{b}\right)\right] \cos (\gamma \zeta) \mathrm{e}^{-\gamma \zeta}-K \operatorname{erfc}\left(\frac{\beta \zeta}{2 T^{1 / 2}}\right) \quad \text { for } t \rightarrow \infty,
$$

$$
K \equiv w_{e}-w_{b}+\frac{A}{\sigma \gamma}
$$

As noted earlier, it is obvious from this solution that if the conditions of the flow just happen to be such that $K \equiv 0$, then the unsteadiness disappears for long time; putting $K$ to zero does, in fact, precisely recover the Thorpe (1987) solution. In the more general case however, $K$ will not be zero, so that it is clear that the layer splits into two distinct parts at long time: an inner layer, of width $(v /(\Omega \cos \alpha))^{1 / 2}$, and an outer layer, whose width turns out to be

$$
\cos \alpha\left(\kappa t^{\star}\left(\frac{\tilde{S} \sigma+1}{1+\tilde{S}}\right)\right)^{1 / 2}
$$

where $t^{\star}$ is a physical time. It is also worth noting that this long-term unsteadiness does not arise in the velocity component up the wall, $u$. This outer-layer structure was found by MacCready \& Rhines (1991), who wrote down a diffusion equation satisfied by $v$, and the diffusion equation had a 'diffusion coefficient' $\kappa(1+\sigma \tilde{S}) /(1+\tilde{S})$. It is evident that the error function segments of the solution are, in fact, solutions of such an equation. However, MacCready \& Rhines (1991) do not seem to have completed the details of the double structure. Actually, to return to a point raised above, if $K \equiv 0$, then it is not immediately obvious that the boundary layer reaches a steady state. What one must do, obviously, to examine such a case is to extend the $s \rightarrow 0$ asymptotic expansions, (7.12) and (7.13), to the next order. In that case, $C_{3}$, for example, behaves like $s^{1 / 2}$, leading to a contribution to $\tilde{w}$, for example, due to the Laplace inversion of $s^{-1 / 2} \exp \left(-\beta \zeta s^{1 / 2}\right)$, which is $\exp \left(-\beta^{2} \zeta^{2} / 4 T\right) /(\pi T)^{1 / 2}$, which does in fact go to zero for all $\zeta$ as $T \rightarrow \infty$, leading to a steady state as asserted.

\subsection{Inverse structure near $s= \pm \mathrm{i}(1+\tilde{S})^{1 / 2}$.}

The above discussion is perhaps premature, since it remains to inquire whether there are other contributions to the long-time behaviour of $(\tilde{u}, \tilde{w}, \tilde{B})$ due to additional singularities near $\operatorname{Re}(s)=0$. Certainly poles, and in some cases branch points, will make contributions to the long-time behaviour of the Laplace inversion; a location on $\operatorname{Re}(s)=0$ where one, or all, of the roots of (7.8) pass through zero would seem likely to lead to such a contribution. Note that at least some of the roots of (7.8) vanish as $s \rightarrow \pm \mathrm{i} \mu$, where $\mu \equiv(1+\tilde{S})^{1 / 2}$. Examination of (7.8) shows that two of the six roots vanish at both locations; the other four are finite. Only half of those roots have a real part that is positive; hence,

and

$$
\left.\begin{array}{ll}
\lambda_{3} \sim a_{o}(s-\mathrm{i} \mu)^{1 / 2}, & a_{o}^{2} \equiv \frac{2 \sigma \mu^{2}}{2 \sigma+\tilde{S}(\sigma+1)} \quad \text { for } \quad s \rightarrow \mathrm{i} \mu, \\
\lambda_{3} \sim a_{o}(s+\mathrm{i} \mu)^{1 / 2} & \text { for } s \rightarrow-\mathrm{i} \mu,
\end{array}\right\}
$$

and, for the two finite roots,

$$
\lambda_{1,2} \sim \mathrm{e}^{\mathrm{i} \pi / 4}\left(\mu\left(1+\frac{1}{2} \sigma\right) \pm\left[\left(1-\frac{1}{2} \sigma\right)^{2} \mu^{2}+\tilde{S}(\sigma-1)\right]^{1 / 2}\right)^{1 / 2}+O(s-\mathrm{i} \mu) .
$$

(For $s \rightarrow-\mathrm{i} \mu$, the asymptotic values for $\lambda_{1}, \lambda_{2}$ are the complex conjugates of those given in (7.22).) Again, what must be done is asymptotic evaluation of the determinant, 
$\Delta$ of (7.12), followed by solution of (7.10). In this case, we find that the two constants $C_{1}, C_{2}$ are finite at $\pm(1+\tilde{S})^{1 / 2}$. Since the roots $\lambda_{1}, \lambda_{2}$ are also non-singular at those locations, the first two terms of the expressions for $\mathscr{L}\{\tilde{u}\}, \mathscr{L}\{\tilde{w}\}, \mathscr{L}\{\tilde{B}\}$ in (7.5)-(7.7) are analytic in this neighbourhood, and therefore contribute nothing to the long-time approximation to the Laplace inversion. However, clearly from (7.21) $\lambda_{3}\left(\bar{\lambda}_{3}\right)$ has a branch point at $\mathrm{i} \mu(-\mathrm{i} \mu)$. After considerable algebra, we find that

$$
\begin{aligned}
C_{3} \sim C_{3 o} \equiv & -\frac{\left(\frac{1}{2} \sigma\right)^{1 / 2}}{\mu} \frac{a_{o}^{2}}{a_{o}(\tilde{S}+2)+\mu^{2}(2 \sigma)^{1 / 2}} \\
& \times\left[\frac{\tilde{S} A}{\mathrm{i} \sigma^{2}}\left(\lambda_{1}+\lambda_{2}\right)-\mu\left(1+\left(\frac{2}{\sigma}\right)^{1 / 2} \frac{1}{a_{o}}\right)\left(v_{e}-v_{b}\right)\right] \quad \text { for } \quad s \rightarrow \mathrm{i} \mu .
\end{aligned}
$$

At the conjugate location, $s=-\mathrm{i} \mu, C_{3} \sim \overline{C_{3 o}}$.

Therefore, inverting, there is a contribution to, say, $\tilde{u}$, which from (7.5) is

$$
\tilde{u}^{(p)} \sim \operatorname{Re}\left(C_{3 o} \mathrm{e}^{\mathrm{i} \mu T}\right) \frac{a_{o} \zeta}{\left(\pi T^{3}\right)^{1 / 2}} \mathrm{e}^{-a_{\sigma}^{2} \zeta^{2} / 4 T},
$$

and from (7.6) and (7.7),

$$
\begin{gathered}
\tilde{w}^{(p)} \sim-\frac{1}{\mu} \operatorname{Im}\left(C_{3 o} \mathrm{e}^{\mathrm{i} \mu T}\right) \frac{a_{o} \zeta}{\left(\pi T^{3}\right)^{1 / 2}} \mathrm{e}^{-a_{o}^{2} \zeta^{2} / 4 T}, \\
\tilde{B}^{(p)} \sim \frac{S}{\mu} \operatorname{Im}\left(C_{3 o} \mathrm{e}^{\mathrm{i} \mu T}\right) \frac{a_{o} \zeta}{\left(\pi T^{3}\right)^{1 / 2}} \mathrm{e}^{-a_{\zeta}^{2} \zeta^{2} / 4 T} .
\end{gathered}
$$

To summarize, the long-time behaviour of the solutions to (7.1)-(7.3) is given by (7.17)-(7.20), apparently appended by (7.24)-(7.26). In the case of the velocity component, $\tilde{w}$, for example, the $O\left(s^{1 / 2}\right)$ term in the approximation to $C_{3}$, as shown in (7.16), leads to a higher-order term in (7.18), of $O\left(T^{-1 / 2}\right)$, which is larger than the order of $\tilde{w}^{(p)}$ in (7.25), which is $O\left(T^{-1}\right)$, for $T \rightarrow \infty$. The same argument holds for $\tilde{B}$. However, for the along-slope velocity component, $\tilde{u}$, the last term in (7.17) is precisely the same order as $\tilde{u}^{(p)}$ in (7.24). Thus, we have,

$$
\left.\begin{array}{rl}
\tilde{u} & \sim \tilde{u}_{o}+\tilde{u}^{(p)}+O\left(T^{-3 / 2}\right), \\
\tilde{w} & \sim \tilde{w}_{o}+O\left(T^{-1}\right), \\
\tilde{B} & \sim \tilde{B}_{o}+O\left(T^{-1}\right) \text { for } \quad T \rightarrow \infty,
\end{array}\right\}
$$

where the subscript notation ( $)_{o}$ refers to the solutions (7.17)-(7.20). Therefore, the decaying periodic contributions due to the branch points at $\pm \mathrm{i} \mu$ are significant for $\tilde{u}$ only. In no case does the contributions from the neighbourhood of the branch points alter the conclusions about the character of the long-time behaviour of the solutions given in $§ 7.1$.

\subsection{Relation to numerical solutions of $\S 3$.}

Since in its initial state the fluid in case (i) of $\$ 3$ is at rest, the linearized analysis provides no insight into that problem; however, there is apparent relevance to the two cases (ii) and (iii), as we shall see below.

We have discussed solutions to the self-similar boundary-layer equations (2.8)-(2.11) with more general edge conditions than those of MacCready \& Rhines (1991), which 
led to (7.1)-(7.3). Let $\tilde{W}_{b}$ denote a generalized angular velocity of the boundary, which is taken to be 1 in the solutions of $\S 3$. Then, we write

$$
\hat{W}=\hat{W}_{e}+\tilde{w}, \quad B^{*}=B_{e}^{*}+2 \hat{W}_{e} \tilde{B}, \quad \tilde{U}=\tilde{u},
$$

and alter the independent variables as

$$
\Theta=-\frac{\zeta}{\left(2 \hat{W}_{e}\right)^{1 / 2}}, \quad t=\frac{T}{2 \hat{W}_{e}} .
$$

With these changes of variable, substitution into (2.9), (2.16) and (2.17), and subsequent linearization (by neglecting products and squares of $\tilde{w}, \tilde{B}, \tilde{v}, \tilde{u}$ ), under the assumption that

$$
\left|\hat{W}_{b}-\hat{W}_{e}\right| \ll 1
$$

we obtain the solutions (7.5)-(7.7), except that the parameter $\tilde{S}$ in the solution must be replaced by

$$
\tilde{S}=\frac{S^{*}-\hat{W}_{e}^{2}}{4 \hat{W}_{e}^{2}} .
$$

We know from the discussion in $\S 5$ that the parabola $S^{*}=\hat{W}_{e}^{2}$ is a boundary between growing-layer and finite-time singularity solutions. That curve crosses $\hat{W}_{e}=1$ at $S^{*}=1$, obviously, so careful examination of the solutions here should give some insight into that transition. That means examining the solutions (7.5)-(7.7) in the vicinity of $\tilde{S}=0$.

\section{Solution near $\tilde{S}=0$}

There is a hint, in results (7.17)-(7.20), of some potential difficulty with the solution at $\tilde{S}=0$ since there is an $\tilde{S}$ in the denominator in (7.17) and (7.18). In fact, note that the expression (7.15) for the determinant $\Delta$ may be rewritten as

$$
\Delta \sim \frac{\mathrm{i} \sigma s(\sigma-1)}{2 \gamma^{3}}\left[\frac{\tilde{S}}{1+\sigma \tilde{S}}+\frac{\beta s^{1 / 2}}{2 \gamma^{3}}\right] .
$$

In establishing (7.17)-(7.19), we considered only the first term in the square brackets of (7.32). However, for $\tilde{S} \ll 1$, we note the appearance of a near-origin pole. (When $\tilde{S}$ is not small, there is no such pole, since the approximate expressions in $\$ 7.1$ require that $|s| \ll 1$.) This new pole is actually on another Riemann sheet for $\tilde{S}>0$, and hence contributes nothing to the long-time behaviour, and (7.17)-(7.19) is recovered. On the other hand, for $\tilde{S}<0$, the pole is in $\operatorname{Re}(s)>0:$ at $s \approx \sqrt{2} \tilde{S}^{2} / \sigma$. All of this is captured in the exact inversion of, say, (7.6), when all of (7.32) is retained,

$$
\begin{aligned}
\tilde{w}=\left[\frac{A}{\gamma \sigma}\left(1-\mathrm{e}^{k^{2} T} \operatorname{erfc}\left(k T^{1 / 2}\right)\right)+\frac{1}{\sigma}\left(w_{e}-w_{b}\right) \mathrm{e}^{k^{2} T} \operatorname{erfc}\left(k T^{1 / 2}\right)\right] \cos (\gamma \zeta) \mathrm{e}^{-\gamma \zeta} \\
-K\left[\operatorname{erfc}\left(\frac{\beta \zeta}{2 T^{1 / 2}}\right)-\mathrm{e}^{k \beta \zeta+k^{2} T} \operatorname{erfc}\left(k T^{1 / 2}+\frac{\beta \zeta}{2 T^{1 / 2}}\right)\right],
\end{aligned}
$$

where $k \equiv 2^{1 / 4} \tilde{S} / \sigma^{1 / 2}$. Similar expressions may be obtained for the other velocity components and $\tilde{B}$. For $T \rightarrow \infty$ with $k>0$, i.e. $\tilde{S}>0$ or, equivalently, $S^{*}>\hat{W}_{e}^{2}$, we recover (7.18) precisely. However, for $T \rightarrow \infty$ but for $k<0\left(S^{*}<\hat{W}_{e}^{2}\right)$, since 
$\operatorname{erfc}(-X)=2-\operatorname{erfc}(X),(7.33)$ takes the limiting form

$$
\begin{array}{r}
\tilde{w} \sim\left[\frac{A}{\gamma \sigma}\left(1+\frac{1}{k(\pi T)^{1 / 2}}-2 \mathrm{e}^{k^{2} T}\right)-\frac{w_{e}-w_{b}}{\sigma \gamma}\left(\frac{1}{k(\pi T)^{1 / 2}}-2 \mathrm{e}^{k^{2} T}\right)\right] \cos (\gamma \zeta) \mathrm{e}^{-\gamma \zeta} \\
-K\left[\operatorname{erfc}\left(\frac{\beta \zeta}{2 T^{1 / 2}}\right)+\frac{1}{k(\pi T)^{1 / 2}} \mathrm{e}^{-\beta^{2} \zeta / 4 T}-2 \mathrm{e}^{k \beta \zeta+k^{2} T}\right] .
\end{array}
$$

Hence, we have an indicator in this linearized analysis of the significance of the locus $S^{*}=\hat{W}_{e}^{2}$ in figure 9 in separating growing-layer solutions from other behaviours. Note that for $\tilde{S} \equiv 0$, the origin singularity is not a pole but a branch point; for this particular value, the temporal growth is algebraic. It is interesting to note that only a Laplace transform procedure like this can uncover such features of the solution; MacCready \& Rhines (1991) found no evidence of this temporal breakdown because they considered only $\tilde{S}>0$ cases.

The above linearized analysis indicates different types of solution on different sides of the curve $S^{*}=\hat{W}_{e}^{2}$. In the region $\hat{W}_{e}>1$ this is not inconsistent with the nonlinear results, although naturally the linearized analysis cannot predict the finitetime singularity, but rather exponential growth. For $\hat{W}_{e}<1$, the situation is slightly more subtle - although the nonlinear calculations lead to steady-state solutions (for $\left.S^{*}>S_{c r i t}^{*}\left(\hat{W}_{e}\right)\right)$, this is not a small perturbation of the trivial solution and consequently cannot be expected to be described by the above linearized analysis.

In terms of other issues of comparison with the numerics, for which we took $\sigma=1$ in many of the computations reported, we need to specialize results (7.27) for $\sigma \equiv 1$. That requires some care. Note by (7.14) that $\beta \equiv 1$ in such a case, for all $\tilde{S}$ values. That means that the final term in (7.17) is identically zero, and elsewhere in (7.17)-(7.20), $\beta$ and $\sigma$ should be set to 1 . In the case of the $\tilde{u}^{(p)}$, in (7.27), note that $a_{o} \equiv 1$, and

$$
C_{3 o}=-\frac{1}{\sqrt{2}} \frac{3 \tilde{S} A \mathrm{e}^{-\mathrm{i} \pi / 4}-(1+\sqrt{2})\left(w_{e}-w_{b}\right)}{1+(1+\sqrt{2}) \mu^{2}} \quad \text { for } \sigma=1 .
$$

The conclusions drawn above about the appearance of the finite-time singularity depend only weakly on $\sigma$, so the conclusion there is unchanged. Finally, we note that there is no qualitative change in the conclusions of this section, or any results quoted, if $\sigma \gg 1$ as in $\S 6$.

\subsection{Significance of the A-term in $K$}

Though equations (7.1)-(7.3) are given here for the boundary layer on a conical surface, they are of course valid for the boundary layer over a surface of arbitrary shape. They are in fact the equations previously obtained by MacCready \& Rhines (1991) for the boundary layer over a planar wall. Whether or not the $A$-term, which represents the gravitationally driven 'slope current', should be retained in $K$, in (7.20), is an important question. If the surface is planar, then some terms neglected in (7.1)-(7.3) are $O(E)$, so the presence of $A$ in the solutions of this section is entirely consistent, as in the discussion of MacCready \& Rhines (1991). However, for a surface with lateral curvature, in particular for the cone configuration explicitly considered in this paper, terms neglected in (7.1)-(7.3) are $O\left(E^{1 / 2}\right)$. Therefore, inclusion of the $A$-term in the solutions (7.14)-(7.17) is not self-consistent, unless in fact $A$ is larger than $O\left(E^{1 / 2}\right)$, namely only for very small slopes, so that the $\cot \alpha$-term in (7.9) is large. Note that discarding $A$ means that there are no steady-state components of the boundary-layer velocity and buoyancy! 
For the conical case in particular, effects due to $A$ must be incorporated through construction of second-order terms in the asymptotic series. The $A$-terms are made even smaller if the Schmidt number, $\sigma$, is large, as it is for a salt/water system. As a practical consequence, therefore, in say spin-up contexts, buoyancy-driven slopecurrent effects are much smaller than the boundary-layer motions due to dynamic forcing through either $v_{e}$ or $v_{b}$, and may therefore be ignored.

In the nonlinear solutions, valid for $r \gg 1$, the equivalent to the parameter $A$ of (7.9) involves an Ekman number, $E$, based on the length scale $r$ rather than the length scale $h$ typical of the overall container geometry; thus, as $r \rightarrow \infty$, this ' $A$ ' $\rightarrow 0$, hence we have taken $\partial B / \partial n=0$ throughout the large-r numerics and analysis of $\S \S 3-4$. Note that in such a case, $K$, from (7.20), is identically zero on the vertical line through $(1,1)$ in figure 9, which corresponds to the Thorpe (1997) solution.

\section{Conclusions}

It has been shown that when a rotating conical container containing a viscous stratified fluid undergoes some abrupt change in conditions (such as rotation rate), then within the framework of the boundary-layer model, a number of diverse flow occurrences are possible. In some cases $\left(S^{*}<S_{c r i t}^{*}\left(\hat{W}_{e}\right), \hat{W}_{e}<1\right)$, a truly steady state is attained after sufficiently long times. Interestingly, there is strong evidence that under some circumstances the 'trivial' state is unstable, in so far as the state of uniform buoyancy and rotation does not (in general) seem to be attained. On this line through the parameter space (i.e. $\hat{W}_{e}=1$ ) a second (nonlinear) state is preferred for $S^{*}<1$. In other cases $\left(\hat{W}_{e}>1, S^{*}<\hat{W}_{e}^{2}\right)$ a finite-time breakdown of the boundary-layer equations may occur. Presumably this leads physically to an eruption of fluid, out of the boundary layer itself; this process appears to be associated with the general retardation of the fluid. In the remaining areas of $\left(S^{*}, \hat{W}_{e}\right)$-space, although a steady state appears to be set up close to the vessel walls, an outer boundary layer also forms, whose thickness grows as the square-root of time. Thus viscous effects will eventually penetrate into the main body of the fluid, which then undergoes a change in flow conditions (both the velocity and the density) in such a way that a steady flow can be sustained, this change occurring over a very long (diffusive) time scale.

In the linearized case considered in $\$ 7$, it is shown that generally the flow splits into two distinct layers: one with $z=O(1)$ and the other with $z=O\left(T^{1 / 2}\right)$. In this case, viscous effects must ultimately diffuse into the main core of the flow, which is thereby modified. However, special circumstances can occur if (in the notation of §7) $K=0$, in which case such unsteadiness does not occur. In this eventuality the effects of viscosity will be confined to the $z=O(1)$ boundary layer, and a fully steady solution to the problem becomes established; this corresponds to the case considered by Thorpe (1987). We have also determined that the linearized solution, which is in general of a quite different character from the nonlinear solutions, is valid only for a narrow range, namely when the difference between the edge and wall azimuthal velocity components is much smaller than $E^{2}$, where $E$ is the Ekman number for the initial rotation.

In this paper we have deliberately restricted our attention to cases without counterrotation, i.e. we have only considered the region $\hat{W}_{e} \geqslant 0, S^{*} \geqslant 0$. However, it is well known (see Bodonyi \& Stewartson 1977; Bodonyi 1978) that even non-stratified counter-rotating flows exhibit complex flow behaviour; the effects of counter-rotation and stratification will be the subject of further investigation.

Finally, we note that, numerically, we can find an interesting phenomenon in 


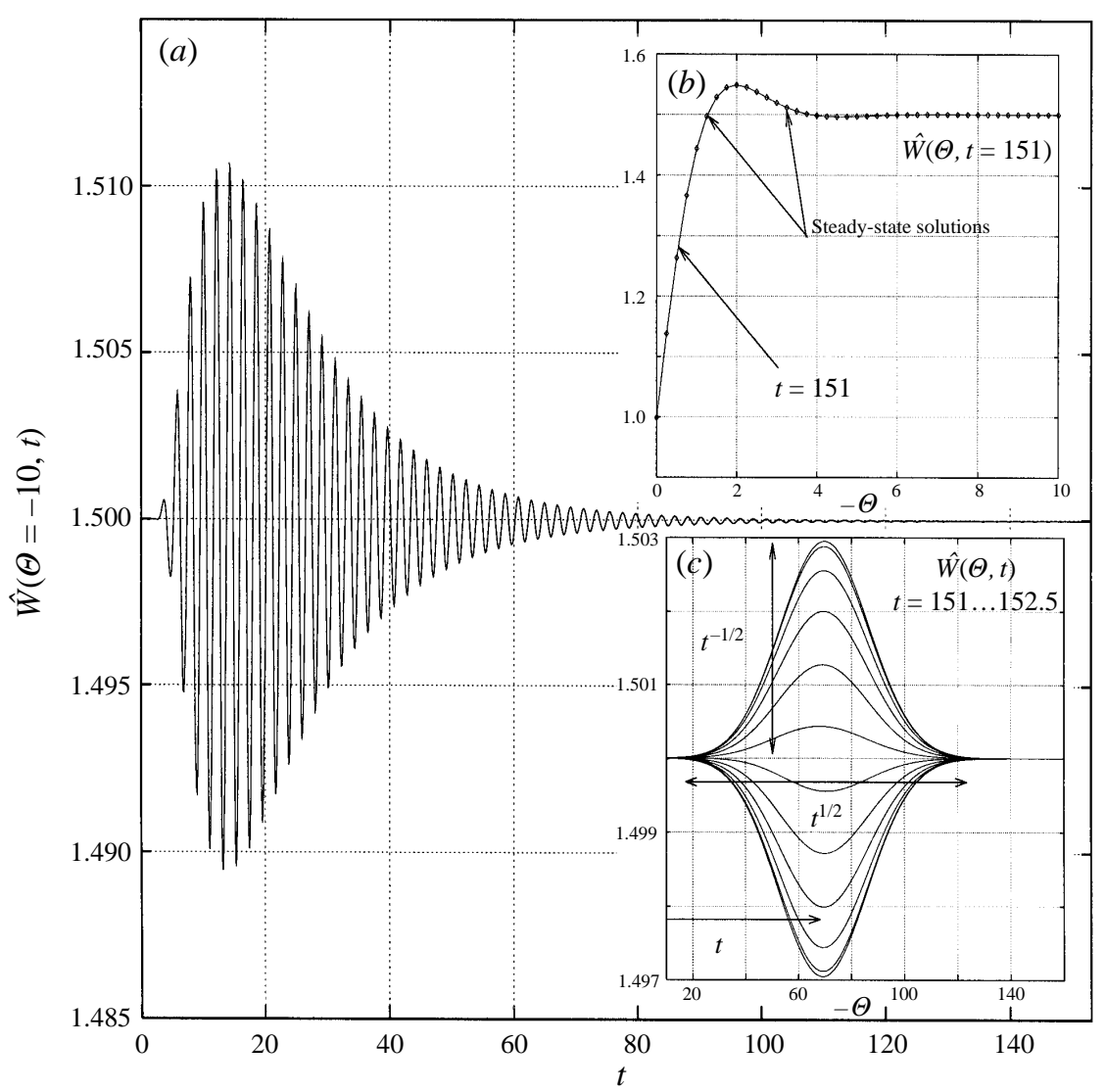

FIGURE 12. Temporal development for $\hat{W}_{e}=1.5, S^{*}=\hat{W}_{e}^{2}$.

areas of the $\left(S^{*}, \hat{W}_{e}\right)$ parameter space that can be associated with the classical (nonstratified) rotating disk problem. In particular, for $S^{*}=\hat{W}_{e}^{2}$ and $\hat{W}_{e}>1$, we note that unsteady calculations do not evolve to any of the steady-state rotating disk solutions. Instead we obtain a solution that, after a sufficiently long time, appears to be the appropriate steady-state solution (from the von Kármán family of similarity solutions) with a superimposed 'wave-packet' part that moves out through the boundary layer linearly with time. As illustrated in figure 12(c), the maximum amplitude of the disturbance decays on a $t^{1 / 2}$ scale, but the length scale also grows like $t^{1 / 2}$, combining to give a sustained oscillatory behaviour in the normal velocity far from the wall. Figure 12(a) shows the evolution of the azimuthal velocity component at a specific point in the boundary layer. Figure 12(b) shows the agreement between the large-time limit of the unsteady solution (here taken at $t=151$ ) and the steady-state solution (from the von Kármán family) in a region near the wall/disk. As is seen from figure $12(c)$ this agreement does not hold as $\Theta$ approaches a value near to $V_{\infty}^{s} t$, where $V_{\infty}^{s}$ is the normal velocity at a point far from the wall/disk, as determined from the appropriate steady-state solution. The frequency of the oscillation is $2 \hat{W}_{e}$, and we believe that it is possible to describe the large-time behaviour asymptotically, in terms of a new variable

$$
\eta^{*}=\frac{\Theta-V_{\infty}^{s} t}{t^{1 / 2}},
$$


but we do not attempt to do so here. This result is in contrast to those presented by Bodonyi (1978) for the unsteady similarity equations of the Bödewadt problem. Although, in a later linear stability analysis, Bodonyi \& Ng (1984) did show that the length scales noted above arise naturally when considering the continuum spectrum in an initial value approach to a perturbation of the similarity solution. However, in their stability analysis the perturbation is shown to decay algebraically. This feature may be of importance when analysing the global spin-up problem for some classes of flows, therefore its description is also a subject for further work.

The support of the EPSRC is gratefully acknowledged. The authors had the benefit of a number of useful discussions with Professor P. A. Davies concerning this work.

\section{REFERENCES}

BöDEwadt, U. T. 1940 Die Drehströmung über festem Grund. Z. Angew. Math. Mech. 20, 241.

BoDONYI, R. J. 1978 On the unsteady similarity equations for the flow above a rotating disc in a rotating fluid. Q. Appl. Math. Mech. 31, 461.

BodonyI, R. J. \& NG, B. S. 1984 On the stability of the similarity solutions for swirling flow above an infinite rotating disk. J. Fluid Mech. 144, 311.

Bodonyi, R. J. \& Stewartson, K. 1977 The unsteady laminar boundary layer on a rotating disk in a counter-rotating fluid. J. Fluid Mech. 79, 669.

Greenspan, H. P. \& Howard, L. N. 1963 On a time-dependent motion of a rotating fluid. J. Fluid Mech. 17, 385.

KÁrmán, T. vON 1921 Über laminare und turbulente Reibung. Z. Angew. Math. Phys. 1, 244.

MacCready, P. \& Rhines, P. B. 1991 Buoyant inhibition of Ekman transport on a slope and its effect on stratified spin up. J. Fluid Mech. 223, 631.

Spence, G. S. M., Foster, M. R. \& Davies, P. A. 1990 The transient response of a contained rotating stratified fluid to impulsively started surface forcing. J. Fluid Mech. 243, 33.

Thorpe, S. A. 1987 Current and temperature variability on the continental slope. Phil. Trans. R. Soc. Lond. A 323, 471.

WALIN, G. 1969 Some aspects of time-dependent motion of a stratified fluid. J. Fluid Mech. 36, 289. 